\title{
Endurance-Aware Cache Line Management for Non-Volatile Caches
}

\author{
JUE WANG, Pennsylvania State University \\ XIANGYU DONG, Qualcomm Technology, Inc. \\ YUAN XIE, Pennsylvania State University \\ NORMAN P. JOUPPI, Hewlett-Packard Labs
}

\begin{abstract}
Nonvolatile memories (NVMs) have the potential to replace low-level SRAM or eDRAM on-chip caches because NVMs save standby power and provide large cache capacity. However, limited write endurance is a common problem for NVM technologies, and today's cache management might result in unbalanced cache write traffic, causing heavily written cache blocks to fail much earlier than others. Although wear-leveling techniques for NVM-based main memories exist, we cannot simply apply them to NVM-based caches. This is because cache writes have intraset variations as well as interset variations, while writes to main memories only have interset variations.

To solve this problem, we propose $\mathrm{i}^{2} \mathrm{WAP}$, a new cache management policy that can reduce both interand intraset write variations. $\mathrm{i}^{2}$ WAP has two features: Swap-Shift, an enhancement based on existing main memory wear leveling to reduce cache interset write variations, and Probabilistic Set Line Flush, a novel technique to reduce cache intraset write variations. Implementing i ${ }^{2}$ WAP only needs two global counters and two global registers. In one of our studies, $\mathrm{i}^{2}$ WAP can improve the NVM cache lifetime by $75 \%$ on average and up to $224 \%$. We also validate that $\mathrm{i}^{2} \mathrm{WAP}$ is effective in systems with different cache configurations and workloads.

Categories and Subject Descriptors: B.3.4 [Memory Structures]: Reliability, Testing, and Fault Tolerance General Terms: Nonvolatile memory, Processors and memory architecture

Additional Key Words and Phrases: Cache, write endurance, wear leveling, interset write variation, intraset write variation, lifetime improvement
\end{abstract}

\section{ACM Reference Format:}

Jue Wang, Xiangyu Dong, Yuan Xie, Norman P. Jouppi. 2014. Endurance-aware cache line management for non-volatile caches. ACM Trans. Architec. Code Optim. 11, 1, Article 4 (February 2014), 25 pages.

DOI: http://dx.doi.org/10.1145/2579671

\section{INTRODUCTION}

Modern chip multiprocessor (CMP) designs tend to use a deeper on-chip cache hierarchy and a larger cache capacity at each level. Today, SRAM and embedded DRAM (eDRAM)

Extension of Conference Paper: This submission is extended from $\mathrm{i}^{2} \mathrm{WAP}$ : Improving Non-Volatile Cache Lifetime by Reducing Inter- and Intra-Set Write Variations published on HPCA13. The additional material provided in the submission includes the background introduction of nonvolatile memory failure mechanisms, a detailed comparison between data movement and data invalidation, sensitivity studies on cache associativity and cache capacity, multiprogramming experiments, and a security threat analysis.

This work is supported in part by SRC grants, NSF 1218867, 1213052, 0903432, and by the DoE under Award Number DE-SC0005026.

Authors' addresses: J. Wang and Y. Xie, Computer Science and Engineering Department, Pennsylvania State University, 111N IST Building, University Park, PA 16802; email: jzw175@cse.psu.edu; X. Dong, Qualcomm Technology, 5775 Morehouse Dr, San Diego, CA 92121; N. Jouppi, Google Inc., 1600 Amphitheatre Parkway Mountain View, CA 94043.

Permission to make digital or hard copies of part or all of this work for personal or classroom use is granted without fee provided that copies are not made or distributed for profit or commercial advantage and that copies show this notice on the first page or initial screen of a display along with the full citation. Copyrights for components of this work owned by others than ACM must be honored. Abstracting with credit is permitted. To copy otherwise, to republish, to post on servers, to redistribute to lists, or to use any component of this work in other works requires prior specific permission and/or a fee. Permissions may be requested from Publications Dept., ACM, Inc., 2 Penn Plaza, Suite 701, New York, NY 10121-0701 USA, fax +1 (212) 869-0481, or permissions@acm.org.

(c) 2014 ACM 1544-3566/2014/02-ART4 $\$ 15.00$

DOI: http://dx.doi.org/10.1145/2579671 
are the common technologies to build on-chip caches. However, neither of them is scalable due to leakage power and cell density concerns. Recently, some emerging nonvolatile memory (NVM) technologies, such as Phase-Change RAM (PCM or PCRAM), Spin-Transfer Torque RAM (STTRAM or MRAM), and Resistive RAM (ReRAM), have been explored as alternative memory technologies. Compared to SRAM and eDRAM, these NVMs have the advantages of high density, low standby power, good process scalability, and nonvolatility. For example, among many ReRAM prototype demonstrations [Waser and Aono 2007; Yang et al. 2008, 2010; Kim et al. 2011; Lin et al. 2010; Tseng et al. 2009; Sheu et al. 2011], a 4MB ReRAM macro [Sheu et al. 2011] can achieve a cell size of $9.5 \mathrm{~F}^{2}$ (15x denser than SRAM) and a random read/write latency of $7.2 \mathrm{~ns}$ (comparable to SRAM caches with the same capacity). Although the write energy of these NVMs is usually tens of picojoules per bit (10x of SRAM write energy, 5x of DRAM), the actual energy saving comes from their nonvolatility. Nonvolatility can eliminate the standby leakage energy, which can be as high as $80 \%$ of the total energy consumption for an SRAM L2 cache [Kim et al. 2003].

Given their energy- and cost-saving potentials (via chip area reduction), on-chip NVM-based cache is an attractive option. Consistent with this expectation, we already see a similar technology shift on the storage and the main memory level. For example, PCM-based storage [Condit et al. 2009; Caulfield et al. 2010; Akel et al. 2011] and main memory [Qureshi et al. 2009b; Lee et al. 2009; Zhou et al. 2009; Qureshi et al. 2009a; Seong et al. 2010a; Ipek et al. 2010; Schechter et al. 2010; Seong et al. 2010b; Yoon et al. 2011] have already been actively explored.

However, it is still challenging to build NVM-based on-chip caches: caches handle much more writes than storage and main memory do, but NVM only has a limited write endurance. For example, PCM can only sustain $10^{8}$ writes before experiencing frequent stuck-at-1 or stuck-at-0 errors [Qureshi et al. 2009a; Ipek et al. 2010; Seong et al. 2010a; Schechter et al. 2010; Seong et al. 2010b; Yoon et al. 2011]. The write endurance of ReRAM is much improved but is still limited at $10^{11}$ [Kim et al. 2011]. For STTRAM, a prediction of up to $10^{15}$ write cycles is often cited, but the best STTRAM endurance test result is less than $4 \times 10^{12}$ cycles [Huai 2008]. This problem of limited write endurance is further amplified by conventional cache management policies: those polices were originally designed for SRAM caches and result in significant nonuniformity in terms of writing to cache blocks, which would cause heavily written NVM blocks to fail much earlier than most other blocks.

There are many wear-leveling techniques to extend the lifetime of NVM main memories [Zhou et al. 2009; Qureshi et al. 2009a; Seong et al. 2010a], but the difference between cache and main memory operational mechanisms makes the existing wearleveling techniques for NVM main memories inadequate for NVM caches. This is because writes to caches have intraset variations in addition to interset variations, while writes to main memories only have interset variations. According to our analysis, intraset variations can be comparable to interset variations for some workloads. This presents a new challenge in designing wear-leveling techniques for NVM caches.

To minimize both inter- and intraset write variations, we introduce $\mathrm{i}^{2} \mathrm{WAP}$ (inter/intraset Write variation-Aware cache Policy), a simple but effective wear-leveling scheme for NVM caches. $\mathrm{i}^{2}$ WAP features two schemes: (1) Swap-Shift is enhanced from the existing main memory wear-leveling techniques and aims to reduce the cache interset write variation; (2) Probabilistic Set Line Flush is designed to alleviate the cache intraset write variation, which is a severe problem for NVM caches and has not been addressed before.

We simulate $\mathrm{i}^{2} \mathrm{WAP}$ with different system configurations, and the result shows that $\mathrm{i}^{2} \mathrm{WAP}$ can reduce the cache write variation by $16 \mathrm{x}$ and improve the lifetime improvement by $75 \%$ (up to $224 \%$ ) compared to state-of-the-art cache management policies. It 
Table I. Area, Performance, and Power Comparison*

\begin{tabular}{|l|c|c|c|c|c|}
\hline & \multicolumn{2}{|c|}{ Volatile Memory } & \multicolumn{3}{c|}{ Nonvolatile Memory } \\
\hline & SRAM & Embedded DRAM & PCM & ReRAM & STTRAM \\
\hline \hline Cell Elements & $6 \mathrm{~T}$ & $1 \mathrm{~T} 1 \mathrm{C}$ & $1 \mathrm{~T} 1 \mathrm{R}$ & Crosspoint & $1 \mathrm{~T} 1 \mathrm{R}$ \\
\hline Cell Area & $140 \mathrm{~F}^{2}$ & $12 \mathrm{~F}^{2}$ & $4.7 \mathrm{~F}^{2}$ & $5 \mathrm{~F}^{2}$ & $16 \mathrm{~F}^{2}$ \\
\hline Read Latency & $70 \mathrm{ps}$ & $0.2 \mathrm{~ns}$ & $<60 \mathrm{~ns}$ & $<10 \mathrm{~ns}$ & $<0.5 \mathrm{~ns}$ \\
\hline Write Latency & $70 \mathrm{ps}$ & $0.2 \mathrm{~ns}$ & $<50 \mathrm{~ns}$ & $<10 \mathrm{~ns}$ & $<0.5 \mathrm{~ns}$ \\
\hline Read Voltage & $0.7 \mathrm{~V}$ & $1.5 \mathrm{~V}$ & $<3 \mathrm{~V}$ & $<0.5 \mathrm{~V}$ & $<1.8 \mathrm{~V}$ \\
\hline Write Voltage & $0.7 \mathrm{~V}$ & $1.5 \mathrm{~V}$ & $<3 \mathrm{~V}$ & $<1 \mathrm{~V}$ & $<1.5 \mathrm{~V}$ \\
\hline Standby Power & Leakage & Refresh & N/A & N/A & N/A \\
\hline
\end{tabular}

*According to ITRS [ITRS 2012].

is effective to both multithread and multiprogram workloads. $\mathrm{i}^{2} \mathrm{WAP}$ only adds $0.25 \%$ performance overhead and negligible hardware overhead, requiring only two global counters and two global registers. Last but not least, we analyze the security feature of $i^{2}$ WAP.

\section{BACKGROUND}

In this section, we first compare three NVM technologies (i.e., PCM, ReRAM, and STTRAM) against conventional SRAM and eDRAM in terms of area, latency, and energy. Moreover, we briefly explain the failure mechanism of each NVM technology.

\subsection{Comparison Between NVM and SRAM/eDRAM}

Compared to SRAM and eDRAM, NVM technologies commonly offer high cell density and zero standby power. For example, a ReRAM prototype [Sheu et al. 2011] was demonstrated to be $15 \mathrm{x}$ denser but with a similar read speed compared to SRAM. Thus, NVM can be used to build larger on-chip caches, reducing cache miss rate and improving performance. Second, NVM caches can reduce energy consumptions. Previous studies have shown that an L2 cache in the Niagara-2 processor consumes more than $20 \%$ of total power consumption [Kim et al. 2013], and leakage energy can be as much as $97 \%$ of the total cache energy consumption [Chandarlapati and Chaudhuri 2007]. Using NVM can eliminate leakage energy when they are standby, hence reducing the total energy consumption.

Table I lists a brief comparison between NVM and SRAM/eDRAM technologies.

\subsection{PCM Technology and Failure Mechanism}

PCM (phase change memory) utilizes the phase-change behavior of chalcogenide alloys (GST) to store data. Every PCM cell contains one GST and one selector transistor. GST can be switched between an amorphous phase (high-resistance, RESET state) and a crystalline phase (low-resistance, SET state) by applying a different voltage pattern. A small voltage is used to sense the data stored in PCM cells.

PCMs have two major failure modes: stuck-RESET and stuck-SET [Kim and Ahn 2005]. Stuck-RESET is caused by void formation or delamination that catastrophically disconnects the electrical path between GST (i.e., the storage element of PCM) and the access device. Stuck-SET is caused by GST aging that makes GST more reluctant to create an amorphous phase after continuously experiencing write cycles, resulting in a degradation of the PCM RESET-to-SET resistance ratio. Both of these failure modes can be commonly observed. ITRS [ITRS 2012] projects that the average PCM write endurance is around $10^{7}-10^{8}$. 


\subsection{ReRAM Technology and Failure Mechanism}

ReRAM is typically based on a solid electrolyte (e.g., oxides) sandwiched by one inert electrode (e.g., Pt) and one electrochemically active electrode (e.g., $\mathrm{Cu}$ or Ag). When there is a positive bias applied on the active electrode, metal ions (e.g., $\mathrm{Cu}^{+}$or $\mathrm{Ag}^{+}$) are formed. These ions then migrate through the solid electrolyte, and they are eventually discharged at the inert electrode, which leads to a growth of dendrite and forms a highly conductive filament in the ON state of the cell. When the applied voltage changes its polarity, an electrochemical dissolution of the filament takes place, and it switches the cell back to the OFF state.

ReRAM has different endurance failure types. One of the them is the anode oxidationinduced interface reaction [Chen et al. 2011]. High temperature, large current/power process, and oxygen ions produced during the forming/SET process cause the oxidation at the anode-electrode interface. Another endurance failure mechanism is the extra vacancy-attributed reset failure effect [Chen et al. 2011]. Electric field-induced extra oxygen vacancy generation during switching may increase the filament size or make the filament rougher, accompanied with the reduced resistance in high-resistance states $\left(\mathrm{R}_{H R S}\right)$ and resistance in low-resistance states $\left(\mathrm{R}_{L R S}\right)$ as well as the increased reset voltage. Recent ReRAM prototypes demonstrate the best write endurance ranging from $10^{10}$ [Lin et al. 2010] to $10^{11}$ [Kim et al. 2011].

\subsection{STTRAM Technology and Failure Mechanism}

STTRAM is the second generation of MRAM (magnetic RAM). STTRAM uses a magnetic tunnel junction (MTJ) to store data. Each MTJ is composed of two ferromagnetic layers. One has a fixed magnetization direction; the other has a free one and can change its direction. The relative direction of these two layers is used to represent a digital " 0 " or " 1 ."

MTJ can be unreliable for two reasons: time-dependent dielectric breakdown (TDDB) and resistance drift [Slaughter et al. 2010]. TDDB is an abrupt increase of junction current owing to a short forming through the tunneling barrier. Resistance drift is a gradual reduction of the junction resistance over time that can eventually lead to reduced read margin. Due to these two issues, the best STTRAM endurance test result so far is less than $4 \times 10^{12}$ [Huai 2008].

\section{INTERSET AND INTRASET WRITE VARIATIONS}

Write variation is a significant concern in designing any cache/memory subsystems with a limited write endurance. Large write variation can greatly degrade the product lifetime because only a small subset of memory cells that experience the worst-case write traffic can result in an entire dead cache/memory subsystem even when the majority of cells are far from wearout.

While the write variation in NVM main memories has been widely studied [Zhou et al. 2009; Lee et al. 2009; Qureshi et al. 2009a, 2009b; Seong et al. 2010a], to our knowledge, the write variation in NVM caches has not. Wear leveling in caches brings extra challenges since there are write count variations inside every cache set (i.e., intraset variations) as well as across different cache sets (i.e., interset variations). In order to demonstrate how severe the problem is for NVM caches, we first do a quick experiment.

\subsection{Definition}

The objective of wear leveling is to reduce write variations and make write traffic uniform. To quantify the cache write variation, we define the coefficient of interset 


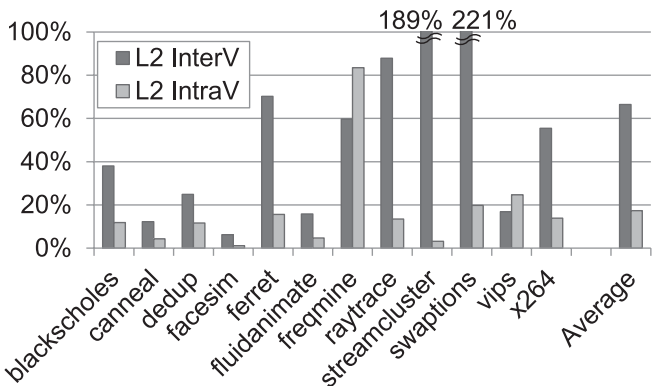

(a)

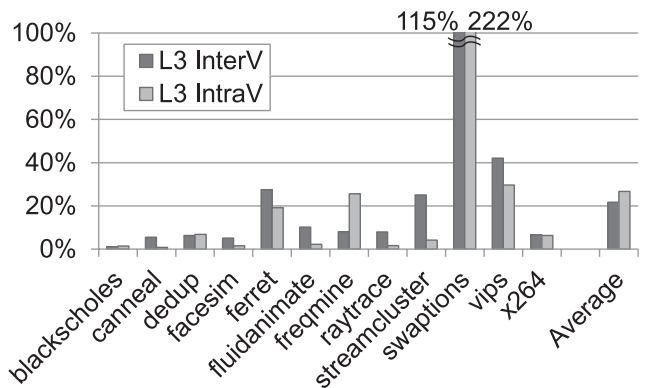

(b)

Fig. 1. The coefficient of variation for interset and intraset write count of L2 and L3 caches in a simulated four-core system with $32 \mathrm{~KB}$ I-L1, 32KB D-L1, 1MB L2, and 8MB L3 caches.

variations (InterV) and the coefficient of intraset variations (IntraV) as follows:

$$
\begin{gathered}
\text { Inter } V=\frac{1}{W_{\text {aver }}} \sqrt{\frac{\sum_{i=1}^{N}\left(\sum_{j=1}^{M} w_{i, j} / M-W_{\text {aver }}\right)^{2}}{N-1}} \\
\text { IntraV }=\frac{1}{W_{\text {aver }} \cdot N} \sum_{i=1}^{N} \sqrt{\frac{\sum_{j=1}^{M}\left(w_{i, j}-\sum_{j=1}^{M} w_{i, j} / M\right)^{2}}{M-1}},
\end{gathered}
$$

where we use Bessel's correction to calculate the standard deviation. $w_{i, j}$ is the write count of the cache line located at set $i$ and way $j$, and $W_{\text {aver }}$ is the average write count:

$$
W_{\text {aver }}=\frac{\sum_{i=1}^{N} \sum_{j=1}^{M} w_{i, j}}{N M},
$$

where $N$ is the number of cache sets, and $M$ is the cache associativity. In short, InterV is the $\mathrm{CoV}$ (coefficient of variation) of the average write count within cache sets; IntraV is the average of the $\mathrm{CoV}$ of the write counts cross a cache set. ${ }^{1}$ If $w_{i, j}$ are all the same, InterV and IntraV are both zero.

Figure 1 shows the experimental results of InterV and IntraV in our simulated fourcore system with $32 \mathrm{~KB}$ eight-way I-L1, 32KB eight-way D-L1, 1MB eight-way L2, and $8 \mathrm{MB}$ eight-way L3 caches. The detailed simulation methodology and the setting are described in Section 8. We compare InterV and IntraV in L2 and L3 caches as we anticipate that NVM will be first used in low-level caches. We observe from Figure 1 that:

(1) Large InterV: Cache lines in different sets might have totally different write frequencies because applications usually have biased memory residency. For instance, streamcluster has $189 \%$ InterV in L2, and swaptions has $115 \%$ InterV in L3. On average, InterV is $66 \%$ in L2 and $22 \%$ in L3.

(2) Large IntraV: A cache line holding hot data might absorb most of the cache write operations, and thus the remaining M-1 lines in the set (for an M-way associative cache) are written less frequently. This causes IntraV. For example, freqmine has

\footnotetext{
${ }^{1}$ We use average $\mathrm{CoV}$ instead of maximum $\mathrm{CoV}$ to keep the definitions of intraset (the CoV of the averages) and interset variations (the average of the CoVs) symmetric.
} 


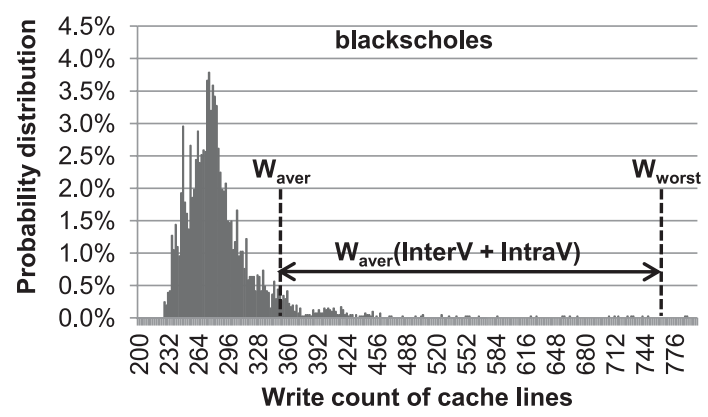

Fig. 2. The L2 cache write count probability distribution function (PDF) of blackscholes.

$84 \%$ L2 IntraV, and swaptions has $222 \%$ L3 IntraV. On average, IntraV is $17 \%$ in L2 and $27 \%$ in L3.

(3) L2 has larger InterV than L3: L2 caches are private for each processor, but all processors share one L3 cache; thus, L3 has a smaller InterV since it mixes different requests. Considering the average write count to L2 is also higher, the larger L2 InterV makes the limited write endurance problem even worse.

(4) IntraV is comparable to InterV: our results show that IntraV is roughly the same or even larger compared to InterV for some workloads. Combining these two types of write variations together significantly shortens the NVM cache lifetime.

\section{CACHE LIFETIME METRICS}

Cache lifetime can be defined in two ways: raw lifetime and error-tolerant lifetime. We define the raw lifetime by the first failure of a cache line without considering any error recovery effort. On the other hand, we can extend the raw lifetime by using error correction techniques and paying overhead in either memory performance or memory capacity [Ipek et al. 2010; Schechter et al. 2010; Seong et al. 2010b; Yoon et al. 2011], and we call it the error-tolerant lifetime. In this work, we focus on how to improve the raw lifetime at first as it is the base of the error-tolerant lifetime. Later, we discuss the error-tolerant lifetime in Section 8.9.

The target of maximizing the cache raw lifetime is equivalent to minimizing the worst-case write count to a cache line. However, it is impractical to obtain the worstcase write count throughout the whole product lifetime, which might span several years. Instead, in this work, we model the raw lifetime by using three parameters: average write count, interset write count variation, and intraset write count variation. The detailed methodology can be described as follows:

(1) The cache behavior is simulated during a short period of time $t_{\text {sim }}$ (e.g., 10 billion instructions on a $3 \mathrm{GHz}$ CPU).

(2) Each cache line write count is collected to get an average write count $W_{\text {aver }}$. Also, we calculate InterV and IntraV according to Equation (1) and Equation (2).

(3) Assuming that the total write variation of a cache line is the summation of its interand intraset variations, ${ }^{2}$ we then have $W_{\text {var }}=W_{\text {aver }} \cdot($ Inter $V+$ IntraV $)$.

(4) The worst-case write count is predicted as $W_{\text {aver }}+W_{\text {var }}$ to cover the vast majority of cases. While it is approximate, Figure 2 validates the feasibility of this approach.

\footnotetext{
${ }^{2}$ InterV and IntraV are not independent, but the worst-case variation can be modeled as the sum of them.
} 


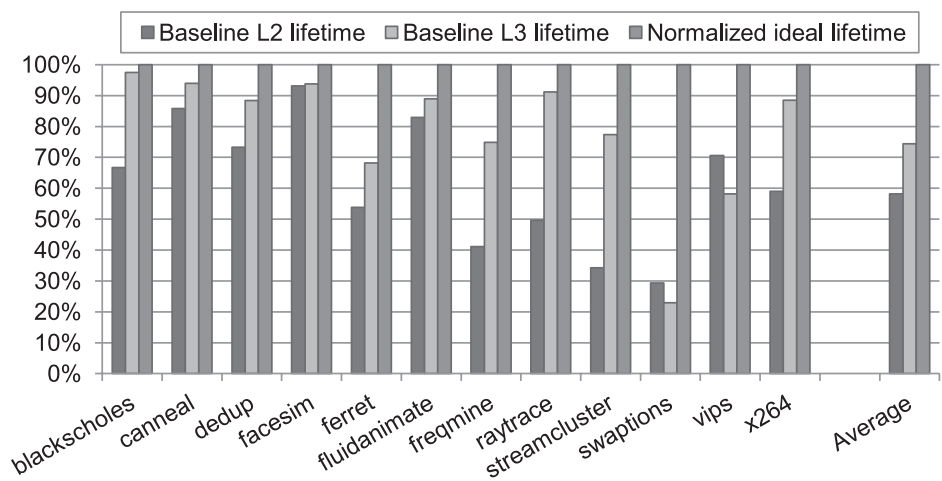

Fig. 3. The baseline lifetime of L2 and L3 caches normalized to the ideal lifetime (no write variations in the ideal case).

(5) Assuming that the general characteristics of cache write operations for one application do not change with time,$^{3}$ the lifetime of the system can be defined as:

$$
t_{\text {total }}=\frac{W_{\max } \cdot t_{\text {sim }}}{W_{\text {aver }}+W_{\text {var }}}=\frac{W_{\max } \cdot t_{\text {sim }}}{W_{\text {aver }}(1+\text { Inter } V+\operatorname{Intra} V)},
$$

where $W_{\max }$ represents the write endurance.

In addition, the lifetime improvement (LI) of a cache wear-leveling technique can be expressed by Equation (5), where $W_{\text {aver_base }}$ and $W_{\text {aver_opt }}$ are the average write count before and after wear leveling, respectively:

$$
L I=\frac{W_{\text {aver_base }}\left(1+\operatorname{Inter} V_{\text {base }}+\operatorname{Intra} V_{\text {base }}\right)}{W_{\text {aver_opt }}\left(1+\operatorname{Inter} V_{\text {opt }}+\operatorname{Intra} V_{\text {opt }}\right)}-1 .
$$

In order to increase LI, we need to reduce InterV and IntraV while not significantly increasing $W_{\text {aver }}$.

We apply the state-of-the-art LRU (least recently used) cache management policy as the baseline, and Figure 3 shows how large the write variation it can cause. Compared to the ideal case where cache writes are evenly distributed, LRU can shorten the cache raw lifetime by $20 \%-30 \%$ under some workloads (e.g., swaptions). If this is the case of future NVM caches, our system will quickly fail even when most of the NVM cells are still healthy. Therefore, it is critical to design a write variation-ware cache management for NVM caches, and we need a cache management policy that can reduce both InterV and IntraV.

\section{STARTING FROM INTERSET WRITE VARIATIONS}

The existing wear-leveling techniques [Zhou et al. 2009; Qureshi et al. 2009a, 2009b; Seong et al. 2010a] focus on increasing the lifetime of NVM main memory. The principle behind these techniques is to introduce an address remapping layer.

\subsection{Challenges in Cache Interset Wear Leveling}

This principle remains the same for cache interset wear leveling, but we should reduce the performance overhead during address remapping since caches are accessed more frequently than main memories.

\footnotetext{
${ }^{3}$ If the system runs different applications over time, the cache write variance can be reduced. However, in this work, we only consider the worst case. It occurs in some practical cases, such as embedded applications in which the data layout could largely remain the same.
} 


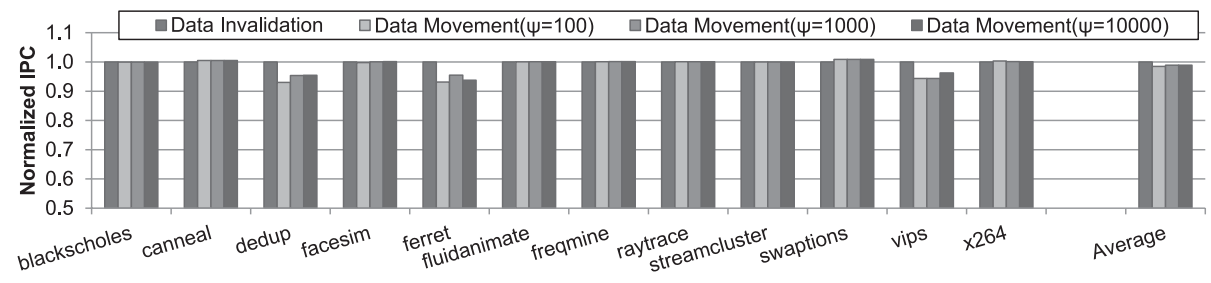

Fig. 4. The performance comparison between data invalidation and data movement.

Using Data Movement: Main memory wear-leveling techniques must use data movement during a remapping because any data loss in main memories is unrecoverable. Moving cache lines from one set to another is costly. First, data movement requires temporary data storage. Second, one cache set movement involves multiple reads and writes, it blocks the cache port, and thus it might cause significant system performance degradation. Start-Gap [Qureshi et al. 2009a] is a recently proposed technique for NVM main memory wear leveling. If we directly extend Start-Gap to handle the cache interset wear leveling, it falls into this category.

Using Data Invalidation: Another option to implement set address remapping for NVM caches is data invalidation. We can use cache line invalidation because we can always restore the cache data later from lower-level memories as long as they are clean. This unique feature of caches provides us a new opportunity to design a low-overhead cache interset wear-leveling technique.

Data invalidation saves the temporary storage and the data movement latency as well. To quantify the performance difference between data movement and invalidation, we use L3 cache interset wear leveling as an example. Equation (6) lists the timing overhead of a cache set movement operation, in which $M$ is cache associativity and $t_{L 3}$ is the L3 access latency (we assume symmetric read/write latency for the sake of simplicity).

$$
t_{\text {move }}=M \times\left(2 \times t_{L 3}\right)
$$

The timing overhead of a cache set invalidation operation is hard to predict precisely since it highly depends on workloads. Equation (7) gives a first-order estimation, where $H i t R$ is the L3 hit rate, WriteR is the L3 write ratio, and $t_{M M}$ is the main memory latency. $t_{\text {invalid }}$ consists of two parts: writing back the dirty data in this set to main memory and restoring data from main memory to L3, which should be hit later. In the first part, $H i t R \times$ WriteR is used to estimate the percentage of dirty blocks, and we assume that the write-back buffer can hide the main memory write latency. In the second part, we assume that the data returned from main memory can be forwarded to L2 before being written into L3.

$$
t_{\text {invalid }}=M \times H i t R \times \text { WriteR } \times t_{L 3}+M \times H i t R \times t_{M M}
$$

We generally have $t_{\text {invalid }}<t_{\text {move }}$ since WriteR is usually small. To further quantify the performance difference between these two, we also simulate two systems, respectively (see Section 8 for detailed simulation settings). In the data movement system, we extend the Start-Gap technique [Qureshi et al. 2009a] and trigger a cache set movement after every $\psi$ cache writes. In the second system, we do not move the cache set but only invalidate it (write back if dirty). Figure 4 shows the performance comparison under different $\psi$ settings (i.e., 100, 1,000, and 10,000). Compared to the data invalidation system, the data movement system has worse performance (i.e., $2 \%$ on average and up to $7 \%$ when $\psi$ equals 100). For the data invalidation system, the performance overhead comes from writing back the dirty data to main memory and restoring data that should be hit later from main memory. The widely used MSHR technique [Kroft 

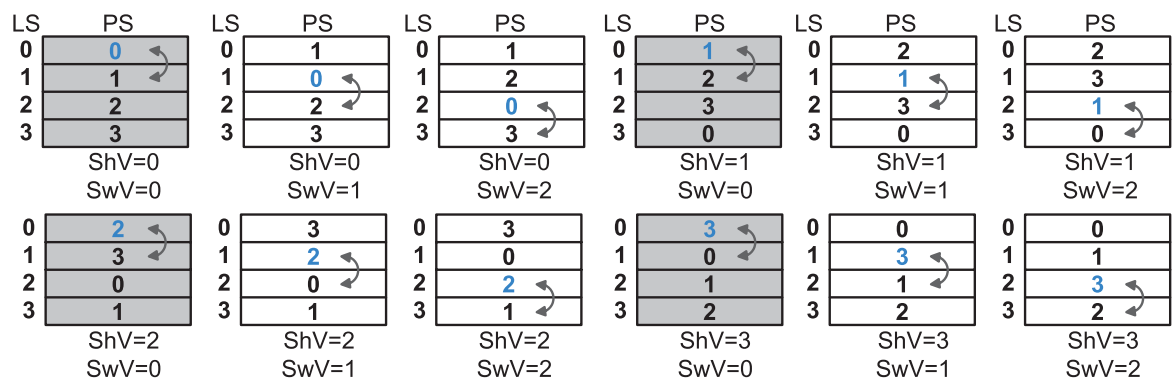

Fig. 5. One SwS shift round in a cache with four sets.

1998] can effectively hide these latencies. However, on the other hand, the performance overhead in the data movement system is always there since we cannot move data in a nonblocking way.

\subsection{Swap-Shift (SwS)}

Considering that data invalidation is more favorable in cache interset wear leveling, we modify the existing main memory wear-leveling technique and devise a new technique called Swap-Shift (SwS).

5.2.1. SwS Architecture. The concept of $\mathrm{SwS}$ is to periodically shift cache set locations. Instead of shifting all the cache sets at once, which hits performance significantly, $\mathrm{SwS}$ only swaps the data of two neighboring sets at once. SwS can eventually shift all the cache sets by one offset after $\mathrm{N}-1$ swaps, where $\mathrm{N}$ is the number of cache sets.

SwS uses a global counter to store the number of cache writes, and we annotate it as $W r$. It also uses two registers, $S w V$ (changing from 0 to $\mathrm{N}-2$ ) and $S h V$ (changing from 0 to $\mathrm{N}-1$ ), to track the current status of swaps and shifts, respectively. SwS has swap round and shift round::

-Swap Round (SwapR): Every time $W r$ reaches a specific threshold (swap threshold, $S T$ ), a swap between cache set $[S w V]$ and set $[S w V+1]$ is triggered. Note that this swap operation only exchanges the set IDs and invalidates the data stored in these two sets (needs write-back if the data are dirty). After that, $S w V$ is incremented by 1. One swap round (SwapR) consists of $\mathrm{N}-1$ swaps and indicates that all the cache set IDs are shifted by 1.

- Shift Round (ShiftR): $S h V$ is incremented by 1 after each SwapR. At the same time, $S w V$ is reset to 0 . One shift round (ShiftR) consists of $\mathrm{N}$ shifts (i.e., SwapR).

Figure 5 is an example of how SwS shifts the entire cache by multiple swaps. It shows the $S w V$ and $S h V$ values during a complete ShiftR, which consists of four SwapRs; it also shows that one SwapR consists of three swaps and all cache sets are shifted by 1 after each SwapR. In addition, after one ShiftR, all cache sets are shifted to the original position and all the logical set indices are the same as the physical ones.

The performance penalty of $\mathrm{SwS}$ is small because only two sets are swapped at once and the swap interval period can be long enough (e.g., million cycles) by adjusting $S T$. The performance analysis of $\mathrm{SwS}$ is in Section 8.8.

5.2.2. SwS Implementation. Figure 6 shows the $\mathrm{SwS}$ implementation. When a logical set number (LS) arrives, the physical set number (PS) can be computed based on three different situations:

(1) If $L S=S w V$, it means that this logical set is exactly the cache set that should be swapped in this ShiftR. Therefore, $P S$ is mapped to the current shift value $(S h V)$. 


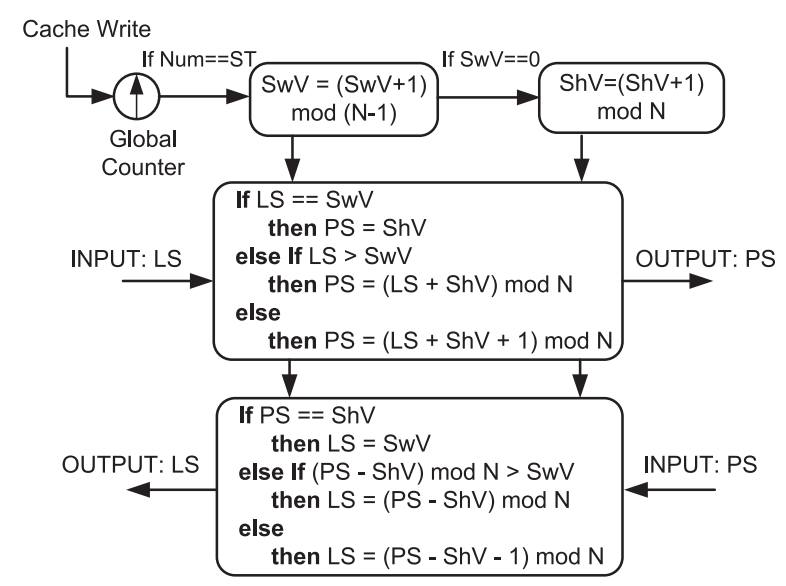

Fig. 6. The mapping between logical (LS) and physical set index (PS) in SwS.

(2) If $L S>S w V$, it means that this set has not been shifted in this ShiftR. Therefore, $P S$ is mapped to $L S+S h V$.

(3) If $L S<S w V$, it means that this set has been already shifted. Therefore, $P S$ is mapped to $L S+S h V+1$.

When a dirty cache line is written back to the next level of cache, the logical set address needs to be regenerated. The mapping from PS to LS is symmetrical and is also given in Figure 6. This mapping policy can be verified by the simple example in Figure 5. Because $S w V$ and $S h V$ are changed along with cache writes, the mapping between LS and PS change all the time. This scheme balances the writes to different physical sets, reducing cache InterV.

Compared to a conventional cache architecture, the set index translation step in SwS only adds a simple arithmetic operation and can be merged into the row decoder. We synthesize the LS-to-PS address translation circuit in a $45 \mathrm{~nm}$ technology, and the circuit can handle an LS-to-PS translation within one cycle under a $3 \mathrm{GHz}$ clock frequency.

\section{INTRASET VARIATION: A MORE SEVERE ISSUE}

SwS only reduces cache interset write variations. Our experiment later in Section 8.2 shows that $\mathrm{SwS}$ alone cannot reduce intraset variations. In this section, we start with two straightforward techniques and then follow with a much improved technique, called PoLF, to tackle the cache intraset variation problem.

\subsection{Set Line Flush}

Intraset write variations are mainly caused by hot data being written more frequently than others. For example, if a cache line is frequently accessed by cache write hits, the corresponding cache set must have a highly unbalanced write distribution.

Traditionally, caches use LRU replacement policy to avoid evicting useful cache lines by marking every accessed block marked toward the MRU (most recently used) position. The LRU policy rarely replaces the hot data that are frequently accessed by cache write hits. This increases the write count of one block and the intraset write variation of the corresponding set.

To solve this problem, we first consider a set line flush (LF) scheme. When there is a cache write hit, LF puts the new data into the write-back buffer directly instead of writing it to the hit data block, and then marks the cache line as INVALID. This process 


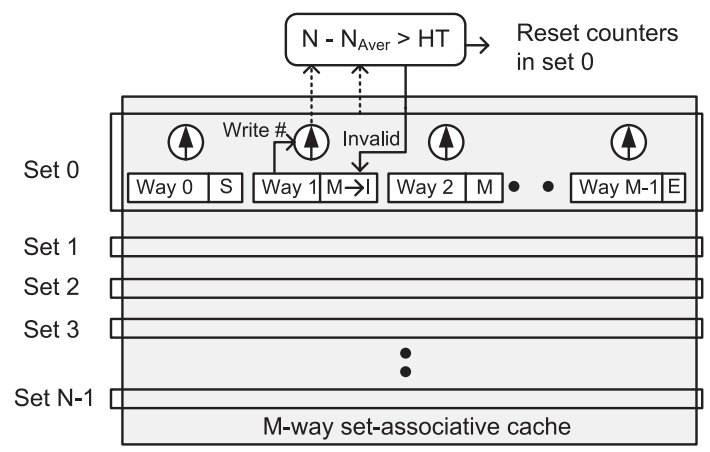

Fig. 7. The cache architecture of HoLF. The counters to store the write count are added to every cache line.

is called set line flush. Using LF, the block containing the hot data has the opportunity to be replaced by other cold data, and the hot data can be reloaded to other cache lines. We invalidate the hot data line instead of moving it to other positions due to the same performance concern explained in Section 5.1.

LF balances the intraset write count, but it flushes data on every cache write hit regardless of data hotness. Obviously, LF greatly harms performance as it evicts useful cache lines every time. Instead, we need a scheme that only flushes hot cache lines that have been heavily written.

\subsection{Hot Set Line Flush}

We can improve the LF scheme by tracking the write count of each cache line and storing this counter in cache tags. We call this enhanced scheme hot set line flush $(\mathrm{HoLF})$. We can detect a hot cache line if its counter is greater than the average value of that cache set by a predetermined threshold, and thus we should flush it. In this way, we can load another data block into this cache line, and reload the hot data into a relatively cold cache line. Figure 7 shows the HoLf architecture.

However, HoLF is still impractical. HoLF adds a large area overhead since it requires one counter for every cache line. Considering that the typical cache line is 64 bytes wide and assuming that the write counter is 20 bit, the hardware overhead is more than $3.7 \%$. HoLF also degrades performance because it updates both maximum and average write counter values in every cache set. It is infeasible to initiate multiple arithmetic calculations for every cache write.

Due to these reasons, we stop the HoLF discussion in this article, and we switch to a further improved solution called PoLF.

\subsection{Probabilistic Set Line Flush}

The key of probabilistic set line flush (PoLF) is to flush hot data probabilistically instead of deterministically.

6.3.1. Probabilistic Invalidation. Unlike HoLF, PoLF only maintains one global counter to count the number of write hits to the entire cache, and it flushes a cache line when the counter saturates regardless of the cache line hotness. Although we cannot guarantee that the hottest data would be flushed, the probability of PoLF selecting a hot data line is high: the hotter the data is, the more likely it will be selected when the global counter saturates. Theoretically, PoLF can still flush the hottest cache line with only one global counter.

Maintaining LRU: Normal LRU policy marks the age bits of the evicted cache line as LRU during a cache line invalidation. However, PoLF should not modify age bits 


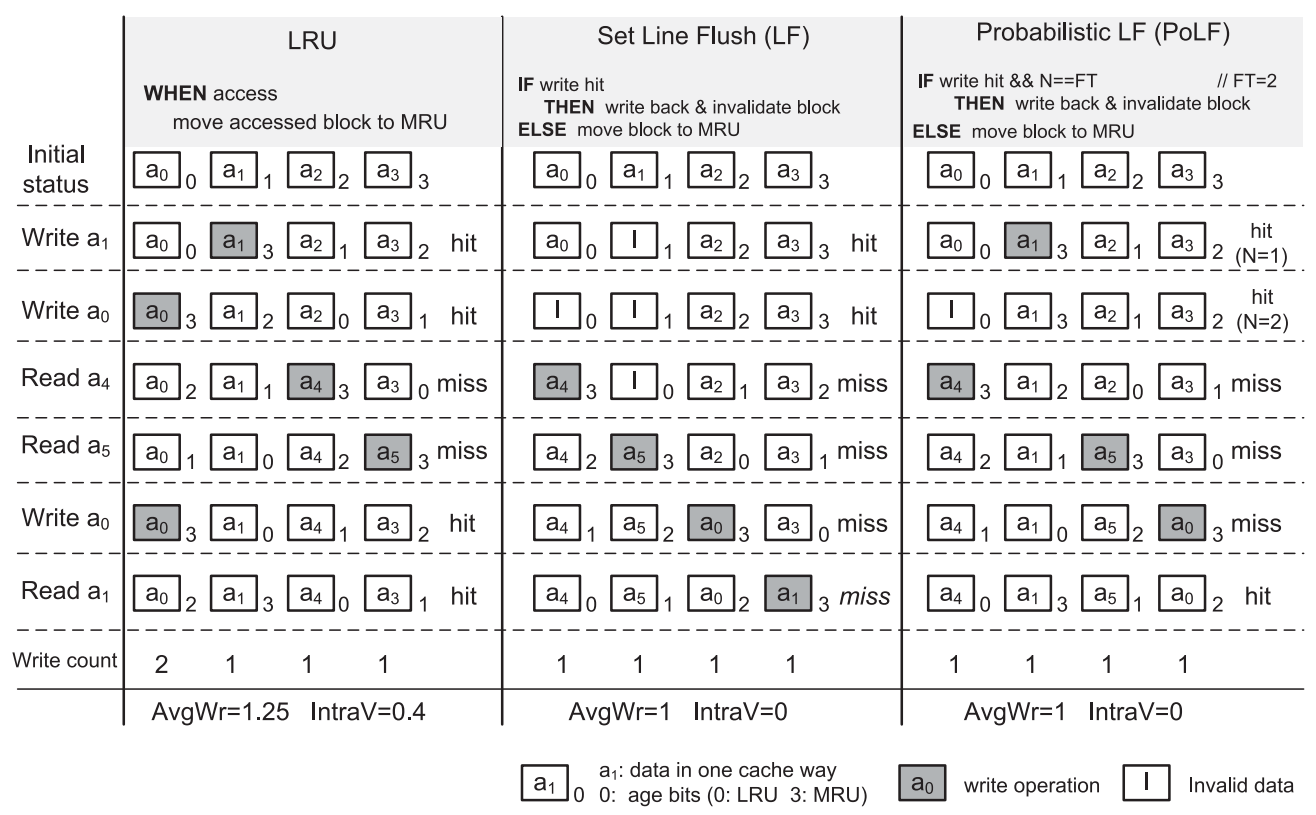

Fig. 8. The behavior of one cache set composed of four ways under LRU, LF, and PoLF policies for the same access pattern. The total write count of each cache way, the average write count, and the intraset variation are marked, respectively.

during a probabilistic invalidation. Otherwise, it is possible that after invalidating a single hot cache line, the same data will be reinstalled in the very same line on a subsequent miss. Therefore, in our design, when PoLF flushes a cache line in response to a probabilistic invalidation, the cache line age bits are unchanged. Later, a subsequent miss will invalidate the actual LRU line and reinstall the hot data in that line. The cache line evicted by probabilistic invalidation remains invalid until it becomes the actual LRU line.

Comparison with Other Policies: Figure 8 shows the behavior of a four-way cache set managed by LRU, LF, and PoLF policies under an exemplary access pattern. We observe that:

(1) For LRU, the hot data $a_{0}$ is moved to the MRU position (age bits $=3$ ) after each write hit and is never replaced by other data. Thus, the intraset variation using LRU is the largest one among all the policies.

(2) For LF, each write hit causes its corresponding cache line to be flushed. The age bits are not changed during write hits. The intraset variation is reduced compared to the LRU policy because the hot data $a_{0}$ is reloaded into another cache line. However, data $a_{1}$ is also flushed since every write hit causes one cache line flush, and it brings one additional access miss.

(3) For PoLF, we let every other write hit cause a cache line flush (i.e., line-flush threshold $F T=2) .{ }^{4}$ Compared to the LRU policy, its intraset variation is reduced because the hot data $a_{0}$ is moved to another cache line. In addition, compared to $\mathrm{LF}$, cache miss rate is reduced because $a_{1}$ is not flushed.

From this example, we can see that PoLF maintains a high probability of replacing a hot cache data and thus reduces IntraV.

\footnotetext{
${ }^{4} F T$ is set as 2 for this illustration. The realistic value is much larger, and we discuss it in Section 8.
} 


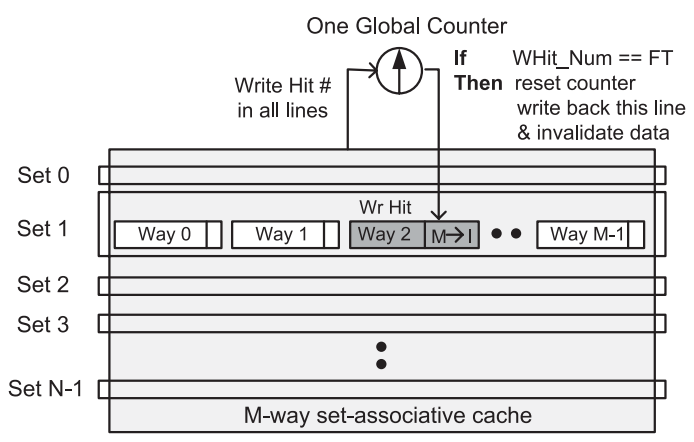

Fig. 9. The cache architecture of PoLF. Only one global write hit counter is added to the entire cache.

6.3.2. PoLF Implementation. Figure 9 shows the PoLF implementation. The only hardware overhead of PoLF is a global counter (one counter for the entire cache) that tracks the total number of write hits to the cache. The counter is only incremented at each write hit event. If the counter saturates at one threshold, then the cache will record the write operation that causes the counter saturation and invalidate the line corresponding to that write hit. The tunable parameter of PoLF is the line-flush threshold $F T$.

\section{I²WAP: PUTTING THEM TOGETHER}

We combine SwS and PoLF together to form our inter- and intraset write variationaware policy, $\mathrm{i}^{2}$ WAP. In $\mathrm{i}^{2} \mathrm{WAP}, \mathrm{SwS}$ and PoLF work independently: SwS reduces InterV, and PoLF reduces IntraV.

The total write variations can be reduced significantly and the product lifetime can be improved. The implementation overhead of $i^{2}$ WAP is:

-One global counter to store the number of write accesses (for $\mathrm{SwS}$ );

- Two registers to store the current cache set swapping and shifting values (for SwS);

-One global counter to store the number of write hits (for PoLF).

\section{EXPERIMENTS}

In this section, we first describe our experiment methodology, then we demonstrate how $\mathrm{SwS}$ and PoLF reduce InterV and IntraV, respectively. Finally, we show how $\mathrm{i}^{2} \mathrm{WAP}$ improves the NVM cache lifetime.

\subsection{Baseline Configuration}

Our baseline is a four-core CMP system. Each core consists of private L1 and L2 caches, and all the cores share an L3 cache. Our experiment makes use of a fourthread OpenMP version of the PARSEC 2.1 [Bienia et al. 2008] benchmark workloads. ${ }^{5}$ We run single applications since they generally represent the worst case. The native inputs are used for the PARSEC benchmark to generate realistic program behavior. We modify the gem5 full-system simulator [Binkert et al. 2011] to implement our proposed techniques and use it to collect cache accesses. Each gem5 simulation run is fast forwarded to the predefined breakpoint at the code region of interest, warmed up by 100 million instructions, and then simulated for at least 10 billion instructions. The characteristics of workloads are listed in Table II, in which WPKI and TPKI are writes and transactions per kilo-instructions, respectively.

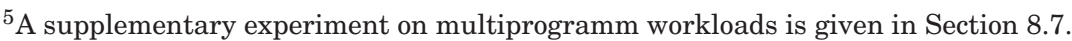


Table II. Workload Characteristics in L2 an L3 Caches Under Our Baseline Configuration

\begin{tabular}{|l|c|c|c|c|c|c|c|c|c|}
\hline & \multicolumn{2}{|c|}{ L2 cache } & \multicolumn{2}{c|}{ L3 cache } & & \multicolumn{2}{c|}{ L2 cache } & \multicolumn{2}{c|}{ L3 cache } \\
\hline Workload & WPKI & TPKI & WPKI & TPKI & Workload & WPKI & TPKI & WPKI & TPKI \\
\hline \hline blackscholes & 0.07 & 0.4 & 0.04 & 0.3 & canneal & 0.04 & 23 & 0.01 & 15 \\
\hline dedup & 1.1 & 4.8 & 0.4 & 0.8 & facesim & 3.3 & 4.7 & 1.1 & 1.4 \\
\hline ferret & 1.8 & 6.3 & 0.2 & 0.5 & fluidanimate & 0.4 & 1.4 & 0.3 & 0.8 \\
\hline freqmine & 1.3 & 6.7 & 0.2 & 0.4 & raytrace & 0.56 & 0.62 & 0.03 & 0.25 \\
\hline streamcluster & 3.7 & 4.2 & 0.9 & 1.1 & swaptions & 1.4 & 2.9 & 0.02 & 0.06 \\
\hline vips & 1.1 & 4.4 & 0.6 & 1.0 & x264 & 0.7 & 16.1 & 0.2 & 0.5 \\
\hline
\end{tabular}

Table III. Baseline Configurations

\begin{tabular}{|l|l|}
\hline System & 4-core, 3GHz, out-of-order CPU model based on ALPHA 21264 \\
\hline \hline SRAM* I-L1/D-L1 caches & $\begin{array}{l}\text { private, 32KB/32KB, 8-way, 64-byte cache line, LRU \& write-back, } \\
\text { write allocate, 2-cycle }\end{array}$ \\
\hline ReRAM L2 cache & $\begin{array}{l}\text { private, } 1 \mathrm{MB}, 8 \text {-way, 64-byte cache line, LRU \& write-back, } \\
\text { write allocate, 30-cycle }\end{array}$ \\
\hline ReRAM L3 cache & $\begin{array}{l}\text { shared, 8MB, 8-way, 64-byte cache line, LRU \& write-back, } \\
\text { write allocate, 100-cycle }\end{array}$ \\
\hline DRAM main memory & 4GB, x16, 8 banks, tRCD-tRP-CL: 11-11-11 \\
\hline
\end{tabular}

*We envision L1 is still SRAM due to performance concerns and NVM write endurance limits.

In this work, we use ReRAM L2 and L3 caches as an example. Our techniques and evaluations are also applicable to other NVM technologies. Table III lists the simulation parameters, and the circuit-level cache parameters (e.g., access latency) are obtained from NVSim [Dong et al. 2012].

\subsection{Effect of SwS on Interset Variation}

The SwS effectiveness in InterV reduction is related to the number of shift rounds (ShiftR). For a cache with $N$ sets, one ShiftR includes $N$ swap rounds (SwapR) and one SwapR has $N-1$ swaps. One ShiftR shifts every cache set through all the possible locations. More ShiftR means better interset wear leveling.

We annotate the round number of ShiftR as RRN:

$$
R R N=\frac{W_{\text {total }}}{S T \times N \times(N-1)}=\frac{W P I \times I_{n}}{S T \times N \times(N-1)},
$$

in which $S T$ is the swap threshold, and $W_{\text {total }}$ is the product of WPI (write access per instruction) and $I_{n}$ (the number of simulation instructions). For the same application, if the execution time is longer, which means $I_{n}$ is larger, we can use a larger $S T$ value to get the same RRN.

To illustrate the relationship between InterV reduction and $R R N$, we run simulations with different configurations and execution lengths. Figure 10 shows the result. As $\mathrm{RRN}$ increases, the cache InterV reduces significantly. When RRN is larger than 100, InterV becomes 95\% smaller. According to Equation (8), this means if we want to make an effective interset wear leveling every month, we can use a relaxed $S T$ value (e.g., 100,000 in this case).

However, simulating a system within a 1-month wall clock time is never realistic. To evaluate the effectiveness of SwS, we use a smaller $S T$ (e.g., ST $=10$ ) in a relatively shorter simulation (e.g., 100 billion instructions) to get a similar RRN. Figure 10(b) shows an L2 InterV reduction after adopting SwS when RRN equals to 100. The average InterV is significantly reduced from $66 \%$ to $1.2 \%$. 

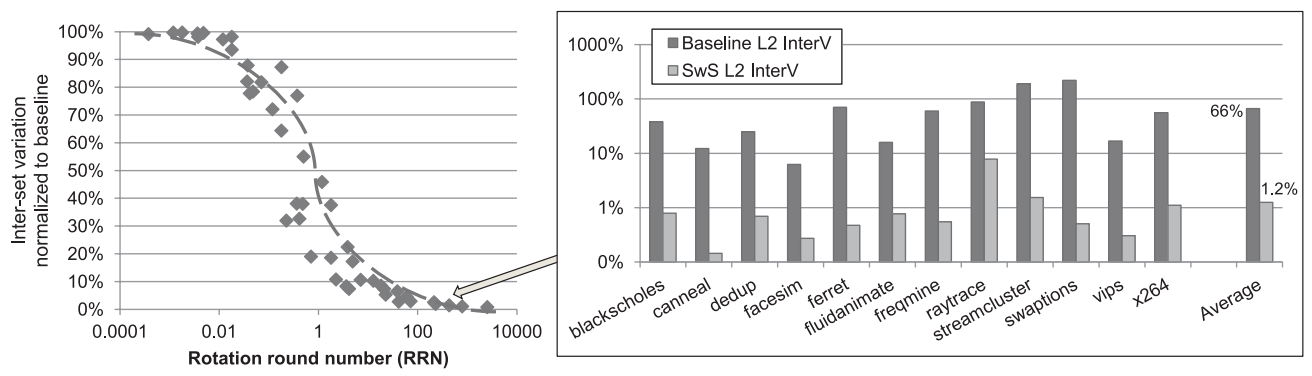

Fig. 10. Interset variations normalized to baseline when RRN increases in SwS scheme. The zoom-in subfigure shows the detailed L2 interset variation of different workloads after adopting the SwS scheme when RRN equals 100 .
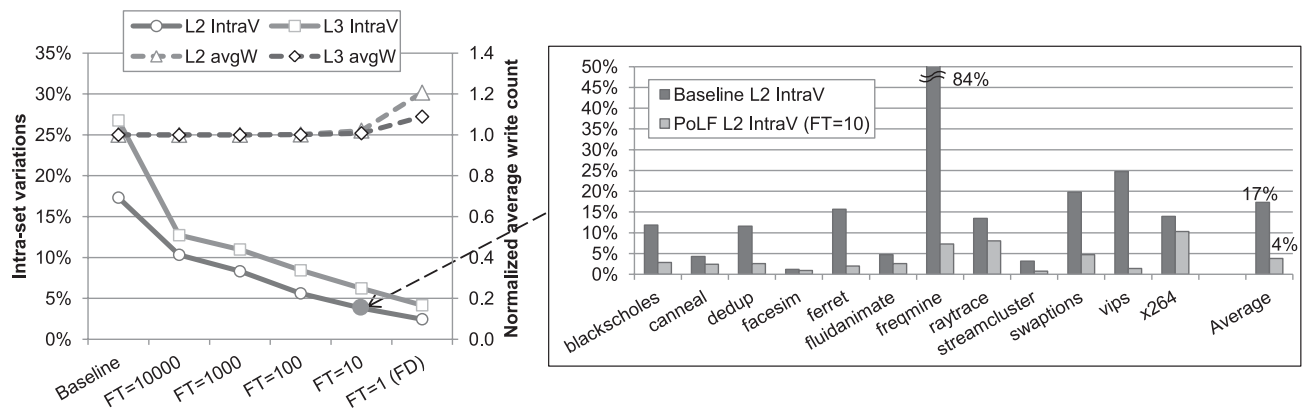

Fig. 11. The average intraset variation and the average write count normalized to baseline for L2 and L3 caches after adopting a PoLF scheme. The zoom-in subfigure shows the detailed L2 intraset variation for different workloads after adopting a PoLF scheme with line-flush threshold (FT) of 10.

In practice, ST can be scaled along with the entire product lifespan since our wearleveling goal is to balance the cache line write count in the scale of several months if not years. Thus, the swap operation in $\mathrm{SwS}$ is infrequent enough to hide its performance impact.

\subsection{Effect of PoLF on Intraset Variation}

Figure 11 shows how PoLF affects IntraV and average write counts for L2 and L3 caches. We can see that PoLF can reduce IntraV significantly and the strength of PoLF can be changed with different $F T$ values.

When FT equals 1, the PoLF scheme flushes every write hit and it is equivalent to the LF scheme. Figure 11 shows that LF can further reduce IntraV compared to PoLF. However, the average write count of LF is increased significantly as well. Thus, considering the impact on both IntraV and average write counts, we choose PoLF with an $F T$ that equals 10 .

The results show that PoLF reduces the average L2 IntraV from $17 \%$ to $4 \%$ and the average L3 IntraV from $27 \%$ to $6 \%$. The average write count is increased by less than $2 \%$ compared to the baseline.

\subsection{Effect of $\mathrm{i}^{2}$ WAP on Total Variation and Lifetime Improvement}

Figure 12 shows the total variations of L2 and L3 caches under different policies. Compared to the baseline, SwS reduces InterV across all the workloads, but it does not reduce IntraV. On the other hand, PoLF reduces IntraV and has a small impact on InterV. By combining SwS and PoLF, $i^{2} W A P$ is able to reduce both InterV and IntraV, 


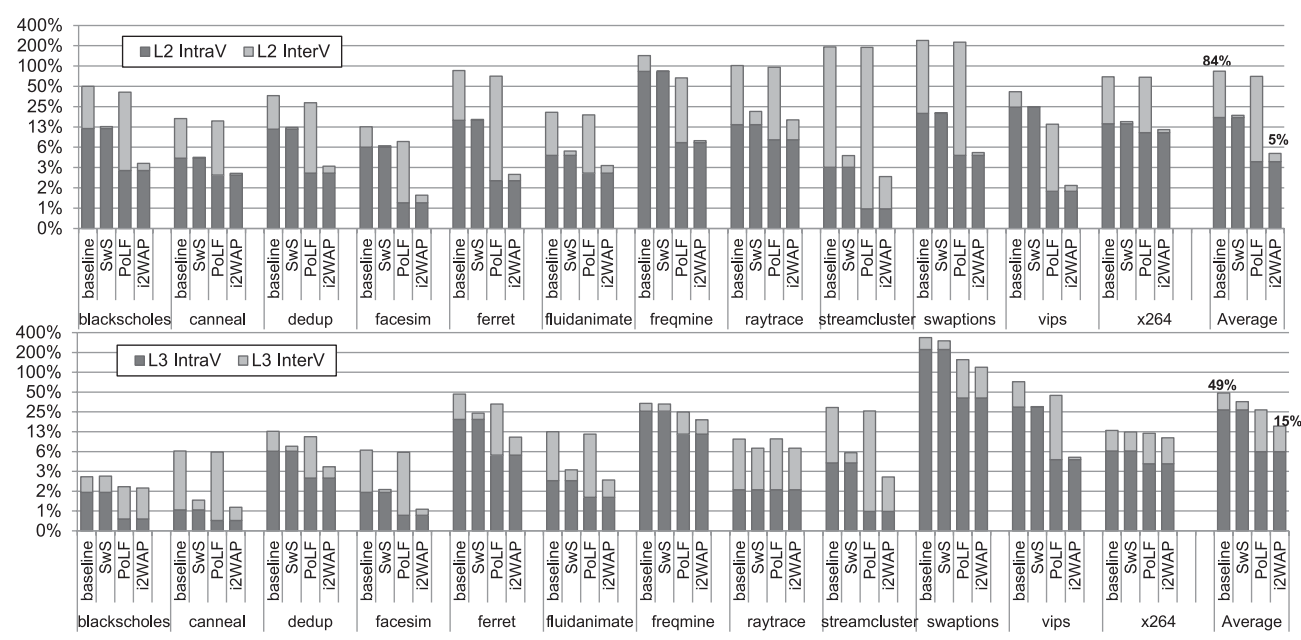

Fig. 12. The total variation for L2 and L3 caches under the baseline configuration, SwS scheme (RRN = $100)$, PoLF scheme $(\mathrm{FT}=10)$, and $\mathrm{i}^{2} \mathrm{WAP}$ policy. Each value is broken down to InterV and IntraV. Note that a log scale is used to cover a large range of variations.
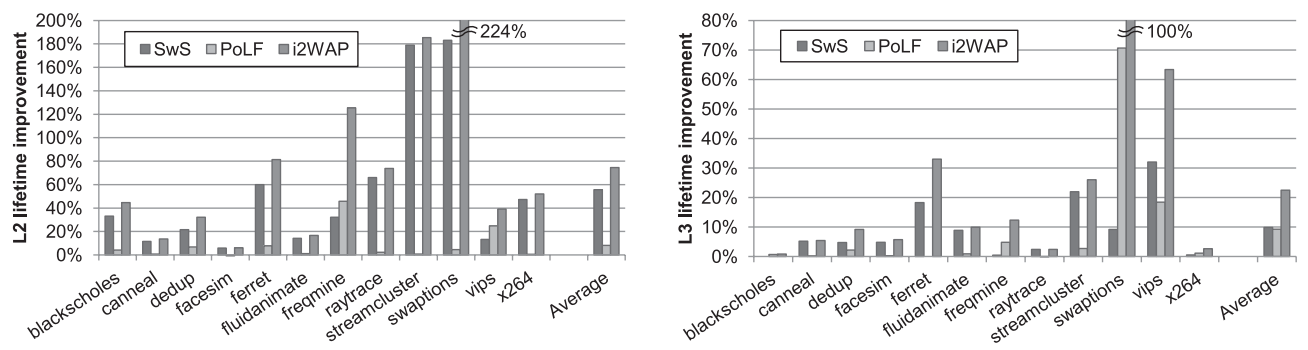

Fig. 13. The lifetime improvement after adopting $\mathrm{i}^{2} \mathrm{WAP}$ using Equation (5). (Left: L2, Right: L3)

evenly distributing writes to every cache line. Figure 12 shows that on average, the total variation is reduced from $84 \%$ to $5 \%$ for L2 caches and from $49 \%$ to $15 \%$ for L3 caches.

Reduced InterV and IntraV mean an improved NVM cache lifetime. Figure 13 shows the lifetime improvement of L2 and L3 caches after adopting SwS only, PoLF only, and the combined $i^{2}$ WAP policy. The lifetime improvement varies based on the workload. Basically, the larger the original variation value is, the bigger the improvement a workload has. The overall lifetime improvement is $75 \%$ (up to 224\%) for L2 caches and $23 \%$ (up to $100 \%$ ) for L3 caches.

\subsection{Sensitivity to Cache Associativity}

As shown in Table III, we use eight-way associative L2 and L3 caches in the baseline system. To study the $\mathrm{i}^{2} \mathrm{WAP}$ effectiveness on different cache configurations, we evaluate its sensitivity to different associativity numbers ranging from four to 32 . All the other system parameters remain the same.

Figure 14 shows the total L2 and L3 variations under different policies when we change the cache associativity. For both L2 and L3 caches in the baseline system, with the increase of the cache associativity, InterV is decreased and IntraV is increased. The reason is that when the cache capacity is fixed, the number of cache sets decreases as the associativity increases. Thus, more writes are merged into one cache set and 

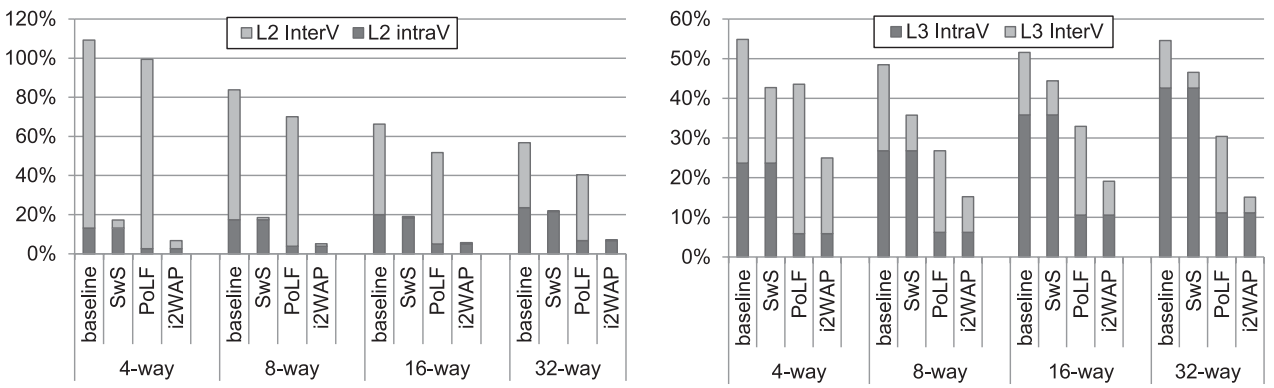

Fig. 14. The total variation for L2 and L3 caches with four-way, eight-way, 16-way, and 32-way under the baseline configuration, SwS scheme $(\mathrm{RRN}=100)$, PoLF scheme $(\mathrm{FT}=10)$, and $\mathrm{i}^{2} \mathrm{WAP}$ policy. Each value is broken down to the interset variation and the intraset variation.
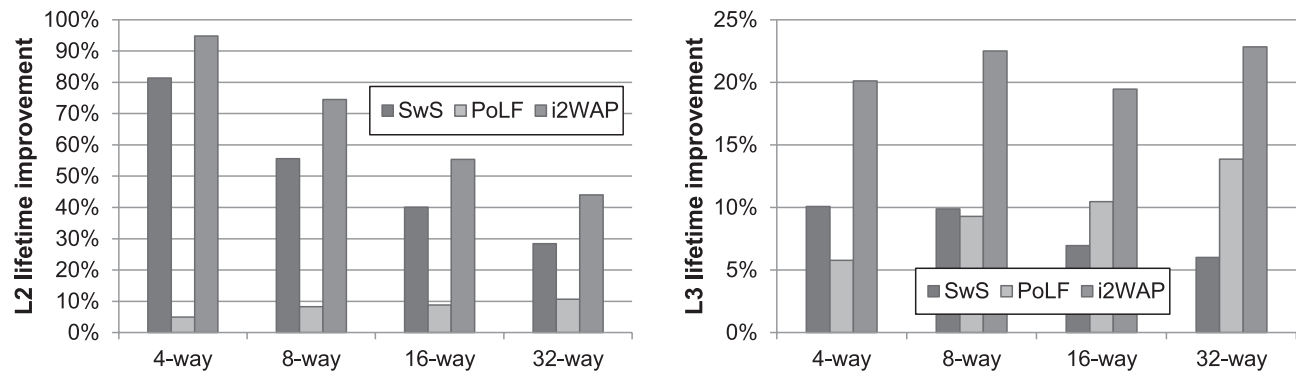

Fig. 15. The lifetime improvement after adopting $\mathrm{i}^{2} \mathrm{WAP}$ with different cache associativities using Equation (5). (Left: L2, Right: L3)

the write variation from one set to another becomes smaller. Furthermore, IntraV is amplified since the number of cache lines in a set is increased and the intraset write imbalance becomes worse.

Regardless of how the associativity changes, adopting $\mathrm{i}^{2} \mathrm{WAP}$ reduces the total variations significantly by combining SwS and PoLF. Figure 14 shows that for the four-way, 16 -way, and 32 -way systems, on average the total variation is reduced from $109 \%$ to $17 \%$, from $66 \%$ to $6 \%$, and from $57 \%$ to $7 \%$ for L2 caches, respectively; for L3 caches, it is reduced from $55 \%$ to $25 \%$, from $52 \%$ to $19 \%$, and from $55 \%$ to $15 \%$, respectively.

Accordingly, Figure 15 shows the lifetime improvement. On average, the lifetime improvement is $95 \%$ and $20 \%$ for L2 and L3, respectively, in a four-way system; it is $55 \%$ and $20 \%$ for L2 and L3, respectively, in an eight-way system; it is $44 \%$ and $23 \%$ for L2 and L3, respectively, in a 16-way system.

\subsection{Sensitivity to Cache Capacity}

We run another sensitivity study on cache capacities. In Section 8, we use 1MB L2 and 8MB L3 caches as shown in Table III. We expect that $i^{2}$ WAP also works effectively on different cache capacities. We conduct experiments on different L2 capacities ranging from $512 \mathrm{kB}$ to $4 \mathrm{MB}$ and different L3 capacities ranging from $4 \mathrm{MB}$ to $32 \mathrm{MB}$. Figure 16 shows the result. On average, the total variation is reduced by $90 \%-95 \%$ for L2 caches and $58 \%-73 \%$ for L3 caches.

Accordingly, Figure 17 shows the lifetime improvement. On average, the lifetime improvement is $66 \%-153 \%$ and $22 \%-26 \%$ for L2 and L3 caches, respectively. These results validate that $\mathrm{i}^{2} \mathrm{WAP}$ works effectively regardless of the cache capacity. For L2 caches, we can see that as capacities increase, the value of lifetime improvement also 

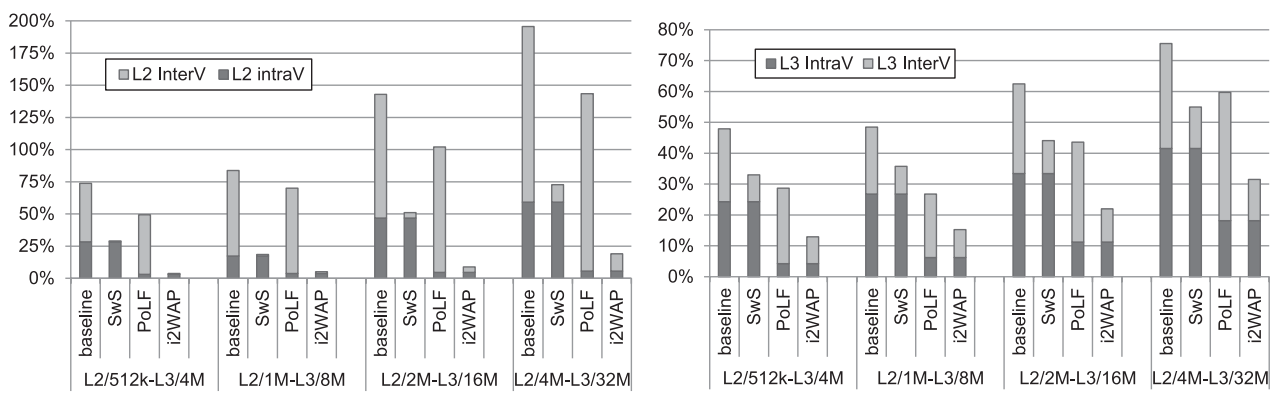

Fig. 16. The total variation for L2 and L3 caches with different capacities under the baseline configuration, SwS scheme $(R R N=100)$, PoLF scheme $(F T=10)$, and $i^{2}$ WAP policy. Each value is broken down to the interset variation and the intraset variation.
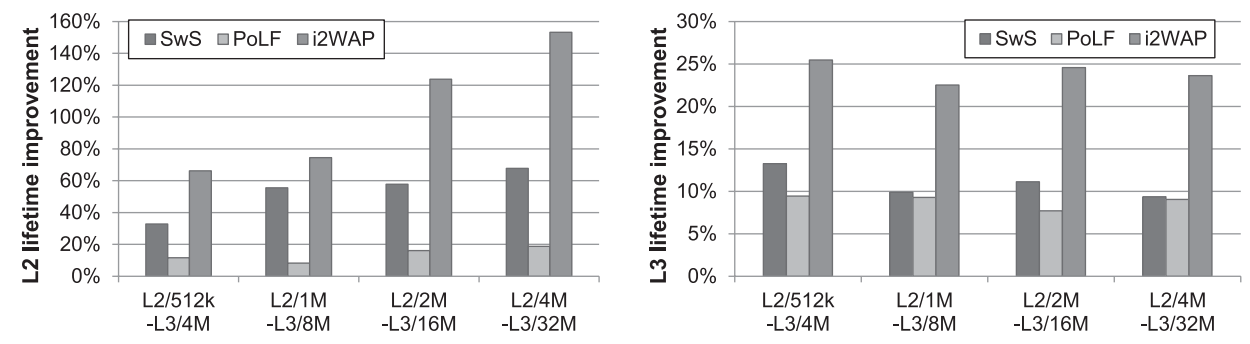

Fig. 17. The lifetime improvement after adopting $\mathrm{i}^{2} \mathrm{WAP}$ with different cache capacities using Equation (5). (Left: L2, Right: L3)

Table IV. The Workload List in the Mixed Groups

\begin{tabular}{|l|c|c|c|}
\hline Mixed Group & Workloads & Mixed Group & Workloads \\
\hline \hline Mix 1 & astar+bwaves+bzip2+gcc & Mix 6 & h264ref+hmmer+milc+namd \\
\hline Mix 2 & bzip2+astar+gobmk+h264ref & Mix 7 & milc+namd+omnetpp+wrf \\
\hline Mix 3 & gromacs+bzip2+gcc+gobmk & Mix 8 & namd+h264ref+gcc+astar \\
\hline Mix 4 & gcc+gromacs+hmmer+namd & Mix 9 & omnetpp+wrf+astar+bwaves \\
\hline Mix 5 & gobmk+h264ref+hmmer+gromacs & Mix 10 & wrf+milc+bwaves+gromac \\
\hline
\end{tabular}

increases. The reason is that the write imbalance is worse in larger-capacity caches, causing a larger variation. Thus, $\mathrm{i}^{2} \mathrm{WAP}$ is more important to large L2 caches. For L3 caches, the variation growth is much flatter than the ones in L2 caches, and IntraV occupies a larger proportion in the baseline. Thus, the L3 cache lifetime improvement is smaller than the one of L2.

\subsection{Sensitivity to Multiprogram Applications}

All the previous simulations are based on multithread workloads. To study the $\mathrm{i}^{2} \mathrm{WAP}$ effectiveness on multiprogram applications, we simulate workload mixtures from the SPEC CPU2006 benchmark suite [SPEC CPU 2006]. The other simulation configurations remain the same as described in Section 8.

Figure 18 shows the variations of L2 and L3 caches for multiprogram workloads in a four-core system. Table IV lists the workload mixtures that we synthesize. Intuitively, multiple cores share one L3 cache and run different programs, and the access traffic to the L3 cache should be well mixed and thus balanced. However, Figure 18 shows that both InterV and IntraV in the shared L3 cache can still be a problem in some cases. On average, InterV and IntraV of L3 caches are 28\% (up to 100\%) and 26\% (up to $64 \%$ ), respectively. Similar to the results of multithread experiments, the variations 


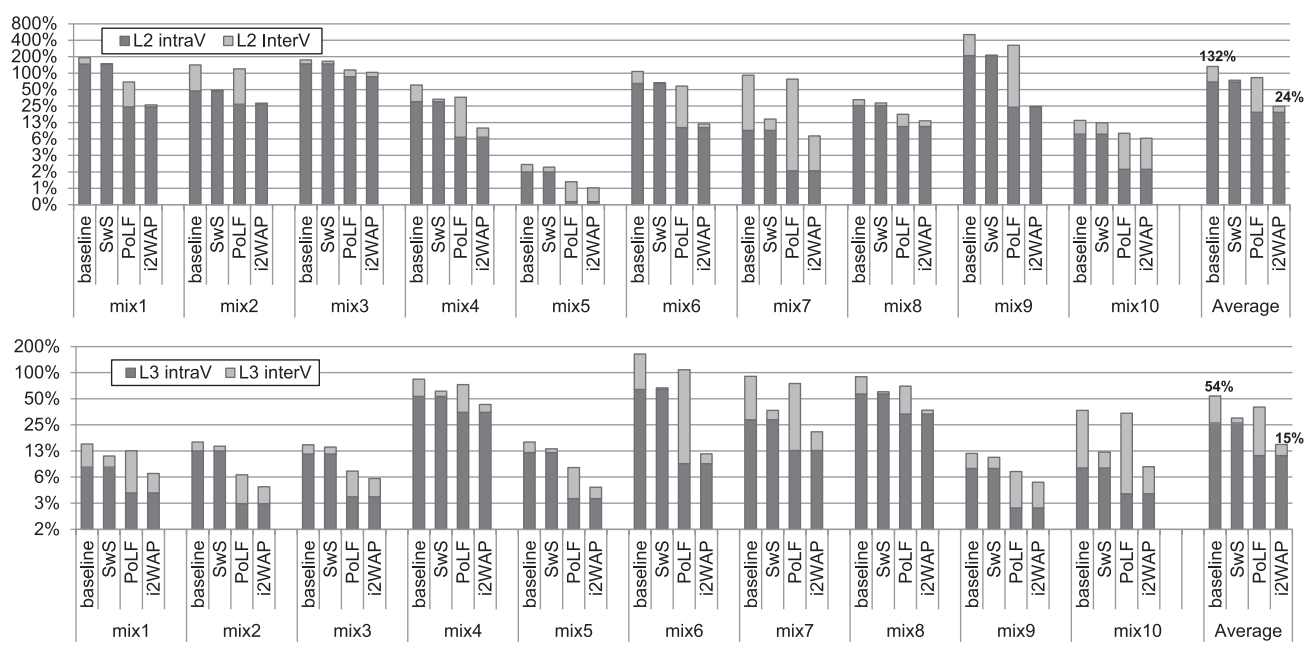

Fig. 18. The total variation for L2 and L3 caches for multiprogram applications using mixed SPEC CPU2006 workloads under the baseline configuration, SwS scheme (RRN $=100)$, PoLF scheme $(F T=10)$, and $i^{2}$ WAP policy. Each value is broken down to the interset variation and the intraset variation.
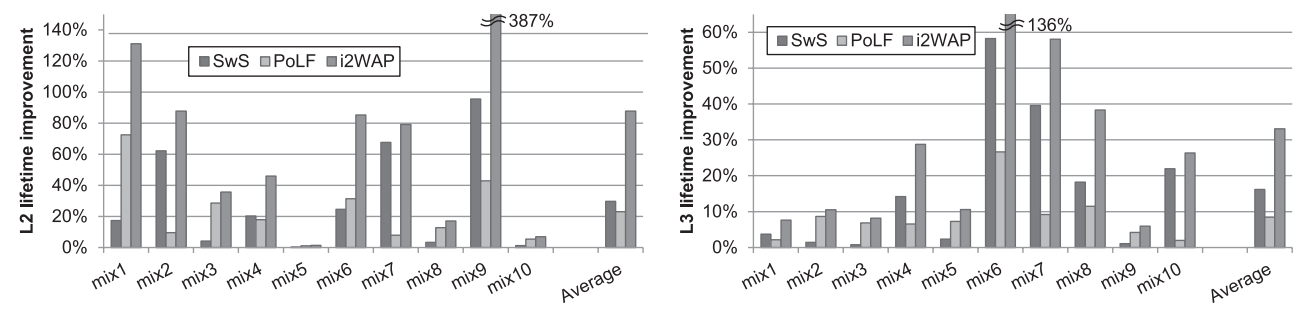

Fig. 19. The lifetime improvement after adopting $\mathrm{i}^{2} \mathrm{WAP}$ for multiprogram applications using Equation (5). (Left: L2, Right: L3)

in L2 caches are larger than the ones in L3 caches, since L2 only serves one program and has a more unbalanced write. On average, the total variation is reduced from $132 \%$ to $24 \%$ for L2 caches and from $54 \%$ to $15 \%$ for L3 caches. Figure 19 shows the lifetime improvement for the multiprogram workloads. For L2 and L3 caches, the overall lifetime improvement is $88 \%$ (up to $387 \%$ ) and $33 \%$ (up to $136 \%$ ), respectively. For the workload mixtures that initially have large InterV, there is a larger space for $\mathrm{i}^{2} \mathrm{WAP}$ to work and improve the lifetime. On the other hand, IntraV is more difficult to reduce since PoLF is based on a probabilistic mechanism, but $\mathrm{i}^{2} \mathrm{WAP}$ still works fairly well for the workload mixtures that initially have large IntraV.

In general, $\mathrm{i}^{2} \mathrm{WAP}$ is effective in reducing cache write variations and improving cache lifetime under multiprogram workloads.

\subsection{Performance Overhead and Impact on Main Memory}

Since $\mathrm{i}^{2} \mathrm{WAP}$ causes extra cache invalidations and extra write-backs on main memory, it is necessary to compare its performance to a baseline system without wear leveling. ${ }^{6}$ To model the contention on the main memory bus, we integrate DRAMSim2 [Rosenfeld et al. 2011], in which open-page policy with FR-FCFS [Rixner et al. 2000] scheduling is

\footnotetext{
${ }^{6}$ The simulator has a protocol to ensure cache coherency when invalidation occurs; thus, the performance overhead of this part is included.
} 


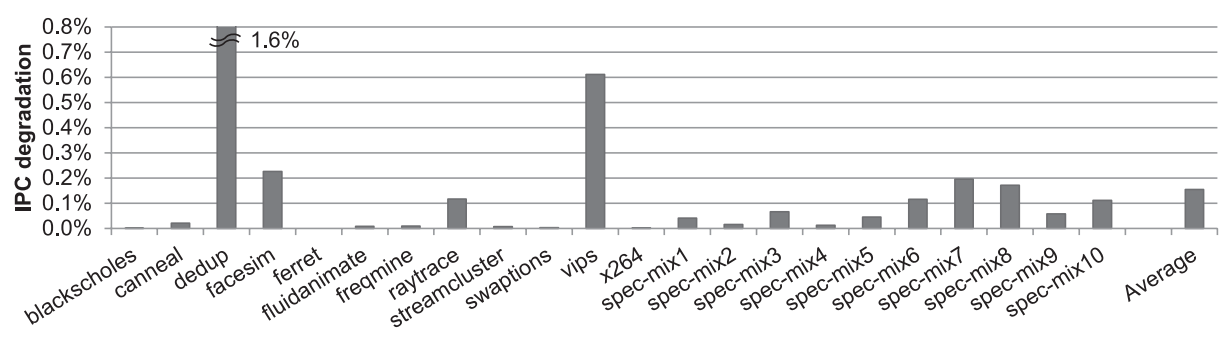

Fig. 20. The system IPC degradation compared to the baseline system after adopting the $\mathrm{i}^{2} \mathrm{WAP}$ policy.

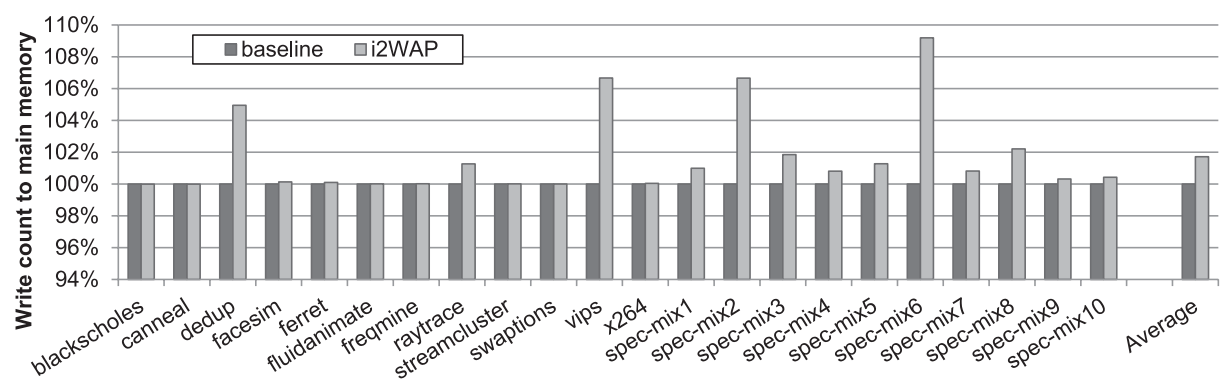

Fig. 21. The write count to the main memory normalized to the baseline system after adopting $\mathrm{i}^{2}$ WAP.

accurately modeled. DRAM timing information was obtained and modified from Micron data sheets [Micron 2011].

Figure 20 shows the performance overhead of a system in which L2 and L3 caches use $\mathrm{i}^{2} \mathrm{WAP}$ with $S T=100,000$ and $F T=10$ compared to a baseline system in which an LRU policy is adopted. Both of the results of the multithread and multiprogram workloads are provided. As shown in Figure 20, on average, the IPC of the system using $\mathrm{i}^{2} \mathrm{WAP}$ is reduced only by $0.15 \%$ compared to the baseline. The performance penalty of $i^{2}$ WAP is very small because of two reasons:

-In SwS, the interval of swap operations is long (e.g., 10 million instructions), and only two cache sets are remapped for each operation.

-In PoLF, write hit accesses are infrequent enough to ensure that the frequency of set

line flush operations is low (e.g., once per $10^{5}$ instructions), and only one cache line is

flushed each time. In addition, designers can trade off between the number of flush operations and the variation value by adjusting $F T$.

Figure 21 shows the write count to the main memory compared to the baseline system after adopting the $\mathrm{i}^{2} \mathrm{WAP}$ policy. The result shows that its impact on the write count is very small, only increasing about $1.7 \%$ on average. For most workloads, the write count is increased by less than $1 \%$. Thus, the impact on memory bus contention is very small. In addition, because most writes can be filtered by caches and the write count of main memory is much smaller than that of caches, the endurance requirement for nonvolatile main memory is much looser.

\subsection{Error-Tolerant Lifetime}

While our analyses are all focused on the raw cache lifetime, this lifetime can be easily extended by tolerating partial cell failures. There are two factors causing the different failure time of cells. The first one is the variation of write counts, which is addressed mainly in this work. The second one is the inherent variation of the cell's lifetime due to process variations, which needs another type of technique to solve (discussed in 


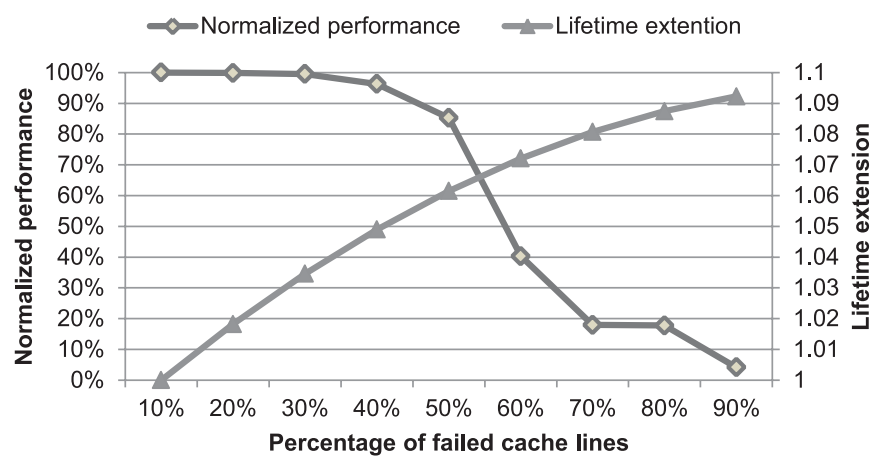

Fig. 22. The performance degradation and lifetime extension during gradual cache line failure on a nonvolatile cache hierarchy.

Section 9). For both factors, the system lifetime can be extended by tolerating a small number of cell failures.

It is much simpler to extend $i^{2} \mathrm{WAP}$ and tolerate the failed cache lines compared to tolerating main memory failures [Ipek et al. 2010; Seong et al. 2010a; Schechter et al. 2010; Seong et al. 2010b; Yoon et al. 2011]. We can force the failed cache lines to be tagged INVALID, so that no further data would be written to the failed cache lines. In this case, the number of ways in the corresponding cache set is only reduced by 1 (e.g., from eight-way associative to seven-way associative).

The error-tolerant lifetime is at least the same as the raw lifetime and may be much longer. However, the performance is degraded because the cache associativity is reduced. Figure 22 shows an analysis of an ReRAM-based cache hierarchy with $32 \mathrm{~KB}$ L1 caches, 1MB L2 caches, and 8MB L3 caches. It shows that if the system can tolerate the failure of $50 \%$ of the cache lines at all levels, the lifetime can be extended by $6 \%$ and the performance penalty is $15 \% .^{7}$

\subsection{Security Threat Analysis}

Thus far, we only consider typical workloads. However, memory technologies with limited write endurance always pose a security threat. An adversary might design an attack that stresses few cache lines to reach their endurance limit and then cause a system failure.

One of the common attacks to wear out NVM is repeated address attack (RAA) [Qureshi et al. 2009a]. Using a simple RAA, an attacker can write a cache line repeatedly and then cause a write endurance failure. It is easy to attack a cache without any endurance-aware policy. A malicious code can attack an L1 data cache by repeatedly writing to one address. Since a cache under normal LRU management never replaces a cache line under a cache hit, the same cache line is continuously overwritten under such an attack. The attacking mechanism is similar for L2 or L3 caches. The only difference is that the attacker would use the higher-level cache's write-back to repeatedly write the lower-level cache. For example, circularly writing $M+1$ ( $M$ is the cache associativity) of data in one L1 cache set would trigger a repeated write pattern to a single L2 cache line. Assuming it takes 10-50 cycles to write back to one L2 cache line,

\footnotetext{
${ }^{7}$ The value of performance degradation and the lifetime extension depend on the cache hierarchy and capacity, but the trends for different configurations are similar.
} 


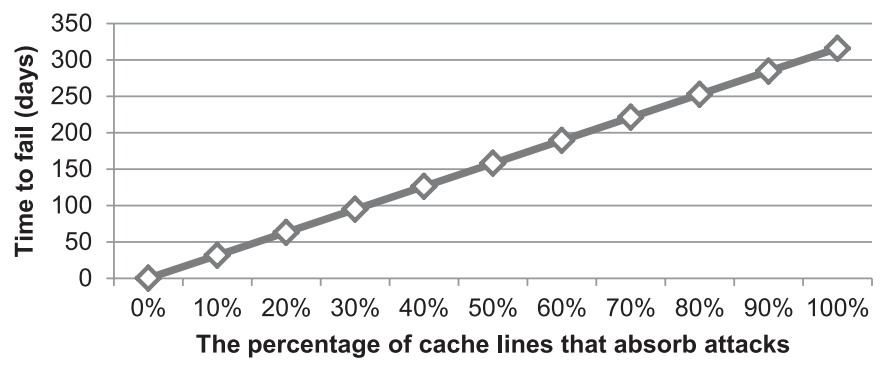

Fig. 23. Relationship between the time to fail and the portion of cache lines absorbing attacks.

the time required to make an L2 cache line failure is:

$$
\text { Time to Fail }=\frac{\text { Cycle per write } \times \text { Cell endurance }}{\text { Cycles per second }}=6-30 \text { minutes } .
$$

Thus, the traditional cache policy opens a serious security problem to NVM caches with limited write endurance.

To defend RAA, we can enhance $\mathrm{i}^{2} \mathrm{WAP}$ by introducing some randomness. The randomized $\mathrm{i}^{2} \mathrm{WAP}$ requires several modifications:

(1) In SwS, we can randomize the swap threshold $S T$ within a predetermined range. Such randomization makes the mapping relationship between physical and logical set IDs unpredictable, therefore increasing the difficulty of RAA.

(2) In PoLF, we can also randomize the line-flush threshold $F T$ within a predetermined range. Cache line invalidation is quickly triggered by repeated write hits. PoLF guarantees a high probability to invalidate the attacked cache line, and data is then loaded in another random location. The threshold randomization makes it more difficult for attackers to predict the new location.

Random number generators have been widely studied [Blum et al. 1986; Kirkpatrick 1981; Qureshi et al. 2009a]. The latency and storage overhead of these generators are small (e.g., one cycle delay with 80 bytes of storage). They can be easily integrated into the $\mathrm{i}^{2} \mathrm{WAP}$ implementation.

The randomized $\mathrm{i}^{2} \mathrm{WAP}$ makes the address remapping layer unpredictable from outside, and it distributes RAA accesses to different cache lines, thus destroying their repetition feature. Figure 23 shows the time to fail for a $1 \mathrm{MB}$ L2 cache when the portion of cache lines mapped from the distributed attacks is increased. The time to make a cache line fail can be extended to several months if an RAA access pattern is distributed to more cache lines. Such a long duration is sufficient to detect an abnormal attack.

\section{RELATED WORK}

There are various previous architectural proposals to extend NVM lifetimes. These prior works can be classified by two basic types of techniques:

The first category focuses on evenly distributing unbalanced write frequencies to all memory lines. Zhou et al. Zhou et al. [2009] proposed a segment swapping policy for PCM main memory. Qureshi et al. proposed fine-grain wear leveling (FGWL) [Qureshi et al. 2009b] and start-gap wear leveling [Qureshi et al. 2009a] to shift cache lines within a page to achieve uniform wearout of all lines in the page. Seong et al. [2010a] addressed potential attacks by applying security refresh. However, this previous work was all focused on extending the lifetime of PCM-based main memory. Other work on NVM caches [Joo et al. 2010] only extended wear-leveling techniques for main memory 
without considering the different operating mechanisms of main memory and caches. As discussed in Section 5.2, these techniques only reduce the interset variation when they are adopted in caches. The cache intraset variation is a new problem and is not considered in previous work.

The second category is about error corrections. The conventional error correction code (ECC) is the most common technique in this category. Dynamically replicated memory [Ipek et al. 2010] reuses memory pages containing hard faults by dynamically forming pairs of pages that act as a single one. Error eorrection pointer (ECP) [Schechter et al. 2010] corrects failed bits in a memory line by recording the position and its correct value. Seong et al. Seong et al. [2010b] propose SAFER to efficiently partition a faulty line into groups and correct the error in the group. FREE-p [Yoon et al. 2011] proposed an efficient means of implementing line sparing. These architectural techniques add different types of data redundancy to solve the access errors caused by limited write endurance. It should be noted that all the techniques from the second category are orthogonal to our proposed policies, and the total system lifetime could be extended further after combining $\mathrm{i}^{2} \mathrm{WAP}$ with them.

Some other works focus on the security threat of nonvolatile main memories [Seznec 2010; Seong et al. 2010a; Qureshi et al. 2009a], and their key idea is to dynamically change the address mapping. Similarly, $\mathrm{i}^{2} \mathrm{WAP}$ can be enhanced by parameter randomization as we discuss in Section 8.10.

\section{CONCLUSION}

Modern computers require large on-chip caches, but the scalability of traditional SRAM and eDRAM caches is constrained by leakage power and cell density. NVM is a promising alternative to build large on-chip caches. However, NVM usually has limited write endurance, and the existing wear-leveling techniques cannot effectively improve the NVM cache lifetime because caches have IntraV in addition to InterV. In this work, we propose $\mathrm{i}^{2} \mathrm{WAP}$, a new endurance-aware cache management policy. $\mathrm{i}^{2} \mathrm{WAP}$ uses $\mathrm{SwS}$ to reduce InterV and PoLF to reduce IntraV, thus improving NVM cache lifetime. To implement $\mathrm{i}^{2}$ WAP, we only need two global counters and two global registers. In one of our experiments, $\mathrm{i}^{2}$ WAP improves the NVM cache lifetime by $75 \%$ on average and up to $224 \%$.

\section{REFERENCES}

A. Akel, A. M. Caulfield, T. I. Mollov, et al. 2011. Onyx: A protoype phase change memory storage array. In Proceedings of the USENIX Conference on Hot Topics in Storage and File Systems. 2-6.

C. Bienia, S. Kumar, J. P. Singh, and K. Li. 2008. The PARSEC benchmark suite: Characterization and architectural implications. In Proceedings of the International Conference on Parallel Architectures and Compilation Techniques. 72-81.

N. Binkert, B. Beckmann, G. Black, et al. 2011. The gem5 simulator. SIGARCH Computer Architecture News 39, 2 (2011), 1-7.

L. Blum, M. Blum, and M. Shub. 1986. A simple unpredictable pseudo-random number generator. SIAM Journal on computing 15, 2 (1986), 364-383.

A. M. Caulfield, A. De, J. Coburn, et al. 2010. Moneta: A high-performance storage array architecture for nextgeneration, non-volatile memories. In Proceedings of the International Symposium on Microarchitecture. 385-395.

J. Chandarlapati and M. Chaudhuri. 2007. LEMap: Controlling leakage in large chip-multiprocessor caches via profile-guided virtual address translation. In Proceedings of International Conference on Computer Design. 423-430.

B. Chen, Y. Lu, B. Gao, et al. 2011. Physical mechanisms of endurance degradation in TMO-RRAM. In Proceedings of the International Electron Devices Meeting. 12.3.1-12.3.4.

J. Condit, E. B. Nightingale, C. Frost, et al. 2009. Better I/O through byte-addressable, persistent memory. In Proceedings of the ACM Symposium on Operating Systems Principles. 133-146. 
X. Dong, C. Xu, Y. Xie, and N. P. Jouppi. 2012. NVSim: A circuit-level performance, energy, and area model for emerging non-volatile memory. IEEE Transactions on Computer-Aided Design of Integrated Circuits and Systems 31, 0 (2012), 994-1007.

Y. Huai. 2008. Spin-transfer torque MRAM (STT-MRAM): Challenges and prospects. Association of Asia Pacific Physical Societies Bulletin 18, 6 (2008), 33.

E. Ipek, J. Condit, E. B. Nightingale, et al. 2010. Dynamically replicated memory: Building reliable systems from nanoscale resistive memories. In Proceedings of the International Conference on Architectural Support for Programming Languages and Operating System. 3-14.

ITRS. 2012. International Technology Roadmap for Semiconductors, Process Integration, Devices, and Structures 2012 Update. Retrieved from http://www.itrs.net/.

Y. Joo, D. Niu, X. Dong, et al. 2010. Energy- and endurance-aware design of phase change memory caches. In Proceedings of the Design, Automation and Test in Europe Conference and Exhibition. 136-141.

C. H. Kim, J.-J. Kim, S. Mukhopadhyay, and K. Roy. 2003. A forward body-biased low-leakage SRAM cache: device and architecture considerations. In Proceedings of the International Symposium on Low Power Electronics and Design. 6-9.

H. Kim, J. H. Ahn, and J. Kim. 2013. Exploiting replicated cache blocks to reduce L2 cache leakage in CMPs. IEEE Transactions on Very Large Scale Integration (VLSI) Systems 21, 10 (2013), 1863-1877.

K. Kim and S. J. Ahn. 2005. Reliability investigations for manufacturable high density PRAM. In Proceedings of the International Reliability Physics Symposium. 157-162.

Y.-B. Kim, S.R. Lee, D. Lee, et al. 2011. Bi-layered RRAM with unlimited endurance and extremely uniform switching. In Proceedings of the Symposium on VLSI Technology. 52-53.

S. Kirkpatrick. 1981. A very fast shift-register sequence random number generator. J. Comput. Phys. 40 (1981), 517-526.

D. Kroft. 1998. Lockup-free instruction fetch/prefetch cache organization. In Proceedings of the International Symposium on Computer Architecture. 195-201.

B. C. Lee, E. Ipek, O. Mutlu, and D. Burger. 2009. Architecting phase change memory as a scalable DRAM alternative. In Proceedings of the International Symposium on Computer Architecture. 2-13.

W. S. Lin, F. T. Chen, C. H. L. Chen, and M.-J. Tsai. 2010. Evidence and solution of over-RESET problem for $\mathrm{HfO}_{x}$ based resistive memory with sub-ns switching speed and high endurance. In Proceedings of the International Electron Devices Meeting. 19.7.1-19.7.4.

Micron. 2011. 4Gb: x4, x8, x16 DDR3 SDRAM. (2011).

M. K. Qureshi, J. P. Karidis, M. Franceschini, et al. 2009a. Enhancing lifetime and security of PCM-based main memory with start-gap wear leveling. In Proceedings of the International Symposium on Microarchitecture. 14-23.

M. K. Qureshi, V. Srinivasan, and J. A. Rivers. 2009b. Scalable high performance main memory system using phase-change memory technology. In Proceedings of the International Symposium on Computer Architecture. 24-33.

S. Rixner, W. J. Dally, U. J. Kapasi, et al. 2000. Memory access scheduling. In Proceedings of the International Symposium on Computer Architecture. 128-138.

P. Rosenfeld, E. Cooper-Balis, and B. Jacob. 2011. DRAMSim2: A cycle accurate memory system simulator. Computer Architecture Letters 10, 1 (2011), 16-19.

S. Schechter, G. H. Loh, K. Straus, and D. Burger. 2010. Use ECP, not ECC, for hard failures in resistive memories. In Proceedings of the International Symposium on Computer Architecture. 141-152.

N. H. Seong, D. H. Woo, and H.-H. S. Lee. 2010a. Security refresh: prevent malicious wear-out and increase durability for phase-change memory with dynamically randomized address mapping. In Proceedings of the International Symposium on Computer Architecture. 383-394.

N. H. Seong, D. H. Woo, V. Srinivasan, et al. 2010b. SAFER: Stuck-at-fault error recovery for memories. In Proceedings of the International Symposium on Microarchitecture. 115-124.

A. Seznec. 2010. A Phase change memory as a secure main memory. Computer Architecture Letters 9, 1 (2010), 5-8.

S.-S. Sheu, M.-F. Chang, K.-F. Lin, et al. 2011. A 4Mb embedded SLC resistive-RAM macro with 7.2ns read-write random-access time and 160ns MLC-access capability. In Proceedings of the International Solid-State Circuits Conference. 200-201.

J. M. Slaughter, N. D. Rizzo, F. B. Mancoff, et al. 2010. Toggle and spin-torque MRAM: Status and outlook. Journal of the Magnetic Society of Japan 5 (2010), 171.

SPEC CPU. 2006. SPEC CPU2006. (2006). http://www.spec.org/cpu2006/. 
Y.-H. Tseng, C.-E. Huang, C.-H. Kuo, et al. 2009. High density and ultra small cell size of Contact ReRAM (CR-RAM) in 90nm CMOS logic technology and circuits. In Proceedings of the International Electron Devices Meeting. 1-4.

R. Waser and M. Aono. 2007. Nanoionics-based resistive switching memories. Nature Materials 6, 11 (2007), 833-840.

J. J. Yang, M. D. Pickett, X. Li, et al. 2008. Memristive switching mechanism for metal/oxide/metal nanodevices. Nature Nanotechnology 3, 7 (2008), 429-433.

J. J. Yang, M.-X. Zhang, J. P. Strachan, et al. 2010. High switching endurance in $\mathrm{TaO}_{x}$ memristive devices. Applied Physics Letters 97, 23 (2010), 232102.

D. H. Yoon, N. Muralimanohar, J. Chang, et al. 2011. FREE-p: Protecting non-volatile memory against both hard and soft errors. In Proceedings of the International Symposium on High-Performance Computer Architecture. 466-477.

P. Zhou, B. Zhao, J. Yang, and Y. Zhang. 2009. A durable and energy efficient main memory using phase change memory technology. In Proceedings of the International Symposium on Computer Architecture. 14-23.

Received April 2013; revised July 2013; accepted October 2013 